\title{
THE SPECTRAL ACTION AND COSMIC TOPOLOGY
}

\author{
MATILDE MARCOLLI, ELENA PIERPAOLI, AND KEVIN TEH
}

In memory of Andrew Lange

\begin{abstract}
The spectral action functional, considered as a model of gravity coupled to matter, provides, in its non-perturbative form, a slow-roll potential for inflation, whose form and corresponding slowroll parameters can be sensitive to the underlying cosmic topology. We explicitly compute the non-perturbative spectral action for some of the main candidates for cosmic topologies, namely the quaternionic space, the Poincaré dodecahedral space, and the flat tori. We compute the corresponding slow-roll parameters and see we check that the resulting inflation model behaves in the same way as for a simply-connected spherical topology in the case of the quaternionic space and the Poincaré homology sphere, while it behaves differently in the case of the flat tori. We add an appendix with a discussion of the case of lens spaces.
\end{abstract}

\section{Contents}

1. Introduction

2. The problem of cosmic topology

2.1. Laplace spectrum and cosmic topology

3. The spectral action and cosmic topology

3.1. The spectral action functional

4. Recalling the case of $S^{3}$

4.1. Euclidean model

4.2. The Poisson summation formula

4.3. The spectral action in 4-dimensions

5. Slow-roll potential from the spectral action

5.1. Nonperturbative corrections and slow-roll potential

5.2. Slow-roll parameters

5.3. Slow-roll parameters for the $S^{3}$-topology

6. The quaternionic cosmology and the spectral action

6.1. The Dirac spectra for $S U(2) / Q 8$

6.2. Trivial spin structure: nonperturbative spectral action

6.3. Nontrivial spin structures: nonperturbative spectral action

6.4. Slow-roll potential and parameters for the quaternionic space

7. Poincaré homology sphere: dodecahedral cosmology

7.1. Generating functions for spectral multiplicities

7.2. The Dirac spectrum of the Poincaré sphere

7.3. The double cover Spin(4) $\rightarrow S O(4)$ 
7.4. The spectral multiplicities $\quad 22$

7.5. The spectral action for the Poincaré sphere 23

7.6. Slow-roll potential in dodecahedral cosmologies 26

8. Flat cosmologies 27

8.1. The spectral action on the flat tori 28

9. Geometric engineering of inflation scenarios via Dirac spectra 31

10. Conclusions 35

11. Appendix: Lens spaces, a false positive 36

11.1. The trouble with the Dirac spectrum on lens spaces 36

11.2. Multiplicities, first case 37

11.3. The spectral action and the Poisson formula

\begin{tabular}{ll|} 
11.4. The other spectrum & 42 \\
\hline
\end{tabular}

11.5. The spectral action for $|D| \quad 46$

11.6. The slow-roll potential: a false positive 48

11.7. Lens spaces: a discrepancy

References 53

\section{INTRODUCTION}

Noncommutative geometry provides models of particle physics, with matter Lagrangians coupled to gravity, based on an underlying geometry which is a product of an ordinary 4-dimensional (commutative) spacetime manifold by a small noncommutative space which determines the matter content of the model. The spectral action functional, which is defined for metric noncommutative spaces (spectral triples) is obtained as the trace of a cutoff of the Dirac operator of the spectral triple by a test function. The asymptotic expansion of the spectral action delivers a classical Lagrangian, which contains gravitational terms (Einstein-Hilbert, conformal gravity, cosmological term) and a coupled matter Lagrangian. For a suitable choice of the noncommutative space the latter recovers the Standard Model Lagrangian and some extensions with right handed neutrinos, and more recently with supersymmetric QCD, see [10], 6].

In trying to understand the cosmological implications of this model, one can work as in [25] with the asymptotic expansion of the spectral action functional, but this only delivers models of the very early universe, near the unification epoch. These can be potentially interesting, as the model contains different possible mechanisms of inflation, related to the presence of effective gravitational and cosmological constants. However, one cannot extrapolate that form of the model towards the modern universe, due to the possible presence of non-pertubative effects in the spectral action at lower energies. The spectral action in its non-perturbative form is typically very difficult to compute exactly. However, the recent result of [9] shows that, for a spacetime whose spatial sections are 3 -spheres $S^{3}$, Wick rotated and compactified to a Euclidean model $S^{3} \times S^{1}$, the spectral action can be 
computed explicitly in non-perturbative form, through a careful use of the Poisson summation formula.

In particular, we show here that the non-perturbative correction observed in [9] for perturbations $D^{2} \mapsto D^{2}+\phi^{2}$ of the Dirac operator gives rise to a slow-roll potential $V(\phi)$ for the field $\phi$, which can be used as a model for inflation. We compute the corresponding slow-roll parameters. These are independent of the artifact of the Euclidean compactification to $S^{3} \times S^{1}$. In particular the dependence on the $\beta$ parameter coming from the size of the $S^{1}$ factor disappears. Moreover, while in the Euclidean compactification, the energy scale $\Lambda$ and the sphere radius $a$ are independent quantities, for a Lorentzian geometry with the Friedmann form of the metric, both the scale factor $a(t)$ and the energy scale $\Lambda(t)$ become time-dependent quantities, related by $\Lambda=1 / a(t)$. Since in the explicit nonperturbative form of the spectral action only the product $\Lambda a$ appears, the resulting slow-roll potential continues to make sense in the Lorentzian signature, and the dependence on the scale factor $a(t)$ disappears through being matched with the $\Lambda=1 / a(t)$ scale. Thus, the slow-roll mechanism obtained from the non-perturbative form of the spectral action can be Wick rotated back to the Lorentzian Friedmann form of the geometry and used as a model from which to derive estimates on cosmological parameters such as the spectral index $n_{s}$ and the tensor-to-scalar ratio.

Thus, we obtain a slow-roll potential for inflation from the non-perturbative corrections to the spectral action, which is potentially sensitive to the geometry and topology of the underlying 3-dimensional sections of spacetime. This is interesting, in the perspective of deriving cosmological signatures of possibly non-simply connected topologies. This is known as the problem of cosmic topology and it has been widely studied by cosmologists in recent years. We review briefly the current state of understanding of this problem and the list of those that are currently considered to the the most likely candidates for non-simply connected cosmic topologies.

Cosmological constraints show that flat or nearly flat, very sightly positively curved, geometries are preferred over negatively curved ones. Combined with requirements of homogeneity on the geometry, this selects as the most likely candidates the flat tori and quotients (Bieberbach manifolds) or the sphere and quotient spherical forms. We compare here the behavior of two among the most promising spherical candidates, the quaternionic space and the Poincaré homology sphere or dodecahedral space, and we show that, in the gravity model based on the spectral action functional, they both behave like the 3 -sphere in terms of the resulting model of inflation with slow-roll potential. We then analyze the case of flat tori, and we show that these instead show a distinctly different behavior in terms of possible models of inflation.

Finally, in an appendix we discuss the case of lens spaces, where a discrepancy in the existing mathematical literature on the Dirac spectrum gives 
rise to a "false positive" in terms of the relation between cosmic topology and inflation.

Our method consists of extending the non-perturbative calculation of the spectral action of the sphere given in [9] to all these other cases, by subdividing the Dirac spectrum into a union of arithmetic progressions with multiplicities that can be interpolated via values of polynomials at points of the spectrum. Then one can apply the Poisson summation formula to each of these progressions and obtain a complete explicit computation of the spectral action non-pertubatively.

\section{The PROBlem of COSMiC TOPOLOGY}

The problem of cosmic topology is the question of whether the spatial topology of the universe can be constrained on the basis of available cosmological data, especially coming from the cosmic microwave background (CMB). A general introduction to cosmic topology is given in [22].

It was known since the mid '90s that the CMB anisotropies may produce constraints on the geometry of the universe [21]. In fact, the constraints on the $\Omega_{0}$ parameter favor a spatial geometry that is either flat or nearly flat, slightly positively curved (see [5]). However, even with the curvature severely constrained by cosmological data, there are still different possible multiconnected topologies that support a homogeneous metric with given nearly flat constant curvature. The curvature constraints thus suggest that spherical space forms $S^{3} / \Gamma$, flat tori $T^{3}$ and Bieberbach manifolds $T^{3} / \Gamma$ are all good possible candidates for cosmic topologies [37. Since the first year of WMAP data [35], the problem of cosmic topology became especially interesting for the main reason that a multiconnected topology may be able to account for some of the anomalies observed in the CMB anisotropies. In fact, the WMAP data suggested possible violations of statistical isotropy in the angular correlation function of the temperature fluctuations. The main anomalies are the quadrupole suppression, the small value of the twopoint temperature correlation function at angles above 60 degrees, and the anomalous alignment of the quadrupole and octupole [36]. These anomalies could be an indication of the presence of interesting (non simply connected) cosmic topologies.

As discussed for instance in 34, there are at present three main approaches to investigating the question of cosmic topology.

- A search for multiple imaging in the CMB sky would reveal the periodicities caused by the matching of sides of a fundamental domain for a manifold that is a compact quotient of a model geometry (flat or spherical). This type of search is knows also as "circles in the sky method".

- A non-trivial cosmic topology is expected to violate the statistical isotropy of the angular power spectrum of CMB anisotropies, that is, the rotational invariance of $n$-point correlations. 
- Different cosmic topologies may also be detectable through correlation patterns of the CMB anisotropy field which may be detectable through the coefficients of the expansion of the field in spherical harmonics.

At present there are no conclusive results that either prove or disprove the presence of a non-simply connected spatial topology in the universe. Although encouraging initial results 24] suggested that one of the most widely studied candidate for cosmic topology, the Poincaré homology sphere or dodecahedral space, could account for the missing large angle correlations of the two-point angular correlation function of the temperature spectrum of the CMB, attempts to account for the quadrupole-octupole alignment in this topology have failed [39]. A "circle in the skies" search based on the first year of WMAP data 12 also failed to identify any non-simply connected topologies.

An explicit description of all the different candidate spherical spaces was given in [16], with an analysis of how they may be detectable through "crystallographic method" through the presence of spikes in the pair separation histogram for three dimensional catalogs of cosmic objects.

In addition to the three approaches mentioned above, there has been recently also an analysis of the problem of cosmic topology from the point of view of residual gravity acceleration, [33. This predicts that, in a non-simply connected topology which is a quotient of either the flat 3-dimensional space or the sphere by a discrete group of isometries, a test particle of negligible mass that feels the gravitational influence of a nearby massive object should also feel a gravitational effect from the translates of the same massive objects in nearby fundamental domains of the group action. This gives rise to a gravitational effect qualitatively similar to dark energy. Due to symmetries, it is shown in 33 that this effect vanishes at first order, but has nontrivial third order effects. In the particular case of the Poincaré homology sphere, it vanishes also at third order and only has non-trivial fifth order effects. It is also shown that, in cases like tori $T^{3}$ with three different translation lengths, the residual gravity acceleration effect tends to pull the space back to its most symmetric form with three equal translation lengths. Thus, cosmology dynamically prefers the most symmetric forms for a given topology.

Recently, predictions of possible cosmic topologies have also been obtained within brane-world scenarios in string theory [26].

2.1. Laplace spectrum and cosmic topology. What is especially interesting from our point of view is the fact that the way possible non-simply connected topologies manifest themselves in the CMB is mostly through properties of the Laplace spectrum of these manifolds.

More precisely, in cosmology it is customary to express the temperature fluctuations of the CMB as a series in the spherical harmonics $Y_{\ell m}$, of the 
form

$$
\frac{\Delta T}{T}=\sum_{\ell=0}^{\infty} \sum_{m=-\ell}^{\ell} a_{\ell m} Y_{\ell m} .
$$

One then looks at the correlation for the parameters $a_{\ell m}$. In the case of $S^{3}$, the off diagonal terms vanish,

$$
\left\langle a_{\ell m} a_{\ell^{\prime} m^{\prime}}^{*}\right\rangle=C_{\ell} \delta_{\ell \ell^{\prime}} \delta_{m m^{\prime}},
$$

while the diagonal terms $C_{\ell}$ give the temperature anisotropy power spectrum. In the case of a spherical space form $S^{3} / \Gamma$, with $\Gamma$ a finite group of isometries, it is well known that these correlation functions in general no longer have vanishing off-diagonal components. The information on the different topologies is therefore encoded in the eigenfunctions of the Laplacian, which replace the usual spherical harmonics of $S^{3}$ in the computation of these correlation functions.

Thus, an explicit computation of the spectrum and eigenfunctions of the Laplacian on the candidate 3-manifolds, as in [23], [31], can be used to produce simulated $\mathrm{CMB}$ skies for the different candidate topologies, which are then compared to the WMAP data for the observed CMB.

Various statistical tests have been developed to compare different candidate topologies and search for a best fit with observational data. In particular, in the case of the simplest spherical geometries, a comparison based on Bayesian analysis was given recently in [28], where simulated CMB maps for these different topologies are also exhibited. The work 28] compares the cosmological predictions of suppression of power at low $\ell$ for five different spherical manifolds: the simply connected case $S^{3}$, the quaternionic space, and the three exceptional geometries, octahedral, truncated cubic, and dodecahedral.

\section{The SPECTRAL ACTION AND COSMiC TOPOLOGY}

In this paper we follow a very different point of view on the problem of cosmic topology. We work within a particular theoretical model of gravity coupled to matter, which arises from the noncommutative geometry models of particle physics developed in [11 and more recently [10. These models are based on extending ordinary spacetime to a product by small extra dimensions, which, unlike in string theory models, are not manifolds but noncommutative spaces.

Within these models, one has a natural choice of an action functional, which is the spectral action of [8]. This is essentially an action functional for gravity on the product space $X \times F$, with $X$ the ordinary 4-dimensional spacetime manifold and $F$ the fiber noncommutative space. The large energy

asymptotic expansion of the spectral action functional delivers a Lagrangian 
with gravity terms including the usual Einstein-Hilbert action with a cosmological term, and additional conformal gravity terms. Moreover, in the asymptotic expansion one also finds a coupled matter Lagrangian, which depends on the choice of the non-commutative space $F$. It is shown in 10 that, for a suitable choice of $F$, the resulting Lagrangian recovers the full Lagrangian of an extension of the minimal Standard Model with right handed neutrinos and Majorana mass terms. More recent work [6] shows that a modified choice of the space $F$ leads to a further extension of the Standard Model that incorporates supersymmetric QCD.

In all these cases, the main feature of these noncommutative geometry models is that one has a non-perturbative action functional on $X \times F$, whose asymptotic expansion delivers a Lagrangian for particle physics coupled to gravitational terms. In other words, gravity on the noncommutative product space $X \times F$ manifests itself as gravity coupled to matter on the ordinary (commutative) spacetime manifold $X$.

3.1. The spectral action functional. The generalization of Riemannian geometry in the world of noncommutative geometry is provided by the notion of spectral triples. These are data $(\mathcal{A}, \mathcal{H}, D)$, with $\mathcal{A}$ the algebra of functions on the (possibly noncommutative) space, $\mathcal{H}$ the Hilbert space of square integrable spinors, and $D$ the Dirac operator. The information corresponding to the metric tensor is encoded in the Dirac operator.

The spectral action functional of $[8]$ is defined as $\operatorname{Tr}(f(D / \Lambda))$, where $\Lambda$ is the energy scale and $f$ is a test function, usually a smooth approximation of a cutoff function. This is regarded as a spectral formulation of gravity in noncommutative geometry. This action functional has an asymptotic expansion at high energies

$$
\operatorname{Tr}(f(D / \Lambda)) \sim \sum_{k \in \operatorname{DimSp}} f_{k} \Lambda^{k} f|D|^{-k}+f(0) \zeta_{D}(0)+o(1),
$$

where $f_{k}=\int_{0}^{\infty} f(v) v^{k-1} d v, f_{0}=f(0), f_{-2 k}=(-1)^{k} \frac{k !}{(2 k) !} f^{(2 k)}(0)$, and integration is given by residues of zeta function $\zeta_{D}(s)=\operatorname{Tr}\left(|D|^{-s}\right)$ at the points $k$ in the Dimension Spectrum, that is, at poles of the zeta function.

It suffices for our purposes to concentrate only on the gravitational sector of the noncommutative geometry model, because that is where we expect to see a signature of cosmic topology to appear. This means that, instead of computing the spectral action on the product space $X \times F$, with a noncommutative space $F$ that accounts for the matter terms in the Lagrangian arising from the asymptotic expansion, we only compute it on the underlying commutative spacetime manifold $X$.

More precisely, in Section 4 we recall the computation for the sphere case done in [9]. In the following sections we obtain explicit non-perturbative computations of the spectral actions on various 3-manifolds that are interesting candidates for cosmic topologies. 
We then show in $\$ 5$ that perturbations of the Dirac operator of the form $D^{2} \mapsto D^{2}+\phi^{2}$, as considered in [9] for the sphere case, provide a slowroll potential for a field conformally coupled to gravity, which provides a mechanism for inflation.

We then compute the spectral action and the corresponding slow-roll parameters explicitly for the various different topologies, two spherical ones and a flat case, and we show that in the spherical cases the slow-roll parameters agree with those for the simply connected topology while in the flat case they are different. We discuss separately the case of lens spaces in the appendix.

Since the spectral action is computed non-perturbatively, the results are not confined to the very early universe near unification energy, but extend to lower energies, so that predictions about slow-roll parameters and cosmological properties like tensor-to-scalar ratio and spectral index can, in principle, be compared with observational data. To obtain a more precise model that can be directly compared with data one should also include further corrections to the slow-roll parameters coming from the additional matter sector (the noncommutative space $F$ ), which will not be considered in this paper.

The main conclusion is that, in models of gravity coupled to matter based on noncommutative geometry and the spectral action functional, there is a coupling of topology and inflation: different spatial topologies can have a measurable effects on the tensor-to-scalar ratio and spectral index, through their effects on the slow-roll parameters of a slow-roll potential coming from perturbations of the Dirac operator and non-perturbative effects in the spectral action functional.

\section{Recalling the CASE of $S^{3}$}

In this section we recall briefly the results of Chamseddine-Connes [9] on the non-perturbative calculation of the spectral action for the 3-sphere, which we need later, when we compare their result to the analogous computation in the cases of the other candidate cosmic topologies.

4.1. Euclidean model. We are interested in investigating cosmological signatures of the spatial topology of the universe. This means the topology of a spatial 3-dimensional section of the 4-dimensional Lorentzian spacetime describing the universe. It is customary, in working with the spectral action functional of noncommutative geometry, to Wick rotate to a Euclidean model of gravity on a compact manifold. Thus, instead of working with a non-compact Lorentzian 4-manifold which is topologically a cylinder $S \times \mathbb{R}$, with $S$ a compact 3-dimensional Riemannian manifold, we Wick rotate and compactify to a Riemannian 4-manifold $X=S \times S^{1}$, where the size of $S$ is unaltered and the size of the compactified $S^{1}$ acquires the meaning of a thermodynamic parameter, an inverse temperature $\beta$, as in [9]. 
The spectral action of a 3 -dimensional manifold $S$ is related in [9] to the 4-dimensional geometry $X=S \times S^{1}$ by showing that the transform

$$
k(x)=\int_{x}^{\infty}(u-x)^{-1 / 2} h(u) d u
$$

relates the spectral action functionals for the 3-dimensional and 4-dimensional geometries by

$$
\operatorname{Tr}\left(h\left(D_{X}^{2} / \Lambda^{2}\right)\right) \sim 2 \beta \Lambda \operatorname{Tr}\left(k\left(D_{S}^{2} / \Lambda^{2}\right)\right),
$$

with $\beta$ the size of the circle $S^{1}$ and the Dirac operator $D_{X}$ of the form

$$
D_{X}=\left(\begin{array}{cc}
0 & D_{S} \otimes 1+i \otimes D_{S^{1}} \\
D_{S} \otimes 1-i \otimes D_{S^{1}} & 0
\end{array}\right),
$$

where $D_{S^{1}}$ has spectrum $\beta^{-1}(\mathbb{Z}+1 / 2)$.

4.2. The Poisson summation formula. The Poisson summation formula states that, for a test function $h$ in Schwartz space $h \in \mathcal{S}(\mathbb{R})$, one has

$$
\sum_{n \in \mathbb{Z}} h(n)=\sum_{n \in \mathbb{Z}} \widehat{h}(n)
$$

or the more general form

$$
\sum_{n \in \mathbb{Z}} h(x+\lambda n)=\frac{1}{\lambda} \sum_{n \in \mathbb{Z}} e^{\frac{2 \pi i n x}{\lambda}} \widehat{h}\left(\frac{n}{\lambda}\right),
$$

with $\lambda \in \mathbb{R}_{+}^{*}$ and $x \in \mathbb{R}$, where $\widehat{h}$ is the Fourier transform

$$
\widehat{h}(x)=\int_{\mathbb{R}} h(u) e^{-2 \pi i u x} d u .
$$

In [9] the Poisson summation formula is applied to a test function of the form $h(u)=P(u) f(u / \Lambda)$, where $P(u)$ is a polynomial function that gives a smooth interpolation for the multiplicites of the Dirac eigenvalues on the 3 -sphere $S^{3}$ and $f$ is a smooth approximation to a cutoff function, used in the spectral action functional. This allows for an explicit nonperturbative computation of the spectral action functional in the case of the 3 -sphere.

As shown in $\S 2.2$ of [9], for a sphere $S^{3}$ with radius $a$ the Dirac spectrum is given by $\pm a^{-1}\left(\frac{1}{2}+n\right)$ for $n \in \mathbb{Z}$, with multiplicity $n(n+1)$. The Poisson summation formula as above gives a spectral action of the form

$$
\begin{aligned}
\operatorname{Tr}(f(D / \Lambda)) & =(\Lambda a)^{3} \widehat{f}^{(2)}(0)-\frac{1}{4}(\Lambda a) \widehat{f}(0)+O\left((\Lambda a)^{-k}\right) \\
& =(\Lambda a)^{3} \int_{\mathbb{R}} v^{2} f(v) d v-\frac{1}{4}(\Lambda a) \int_{\mathbb{R}} f(v) d v+O\left((\Lambda a)^{-k}\right),
\end{aligned}
$$

where $\widehat{f}^{(2)}$ denotes the Fourier transform of $v^{2} f(v)$. 
4.3. The spectral action in 4-dimensions. The corresponding computation of the spectral action for $S^{3} \times S^{1}$ is done in [9] using Poisson summation

$$
\sum_{(n, m) \in \mathbb{Z}^{2}} g\left(n+\frac{1}{2}, m+\frac{1}{2}\right)=\sum_{(n, m) \in \mathbb{Z}^{2}}(-1)^{n+m} \widehat{g}(n, m),
$$

for a function of two variables

$$
g(u, v)=2 P(u) h\left(u^{2}(\Lambda a)^{-2}+v^{2}(\Lambda \beta)^{-2}\right),
$$

where $P(u)$ is the polynomial that interpolates the multiplicities of the spectrum on the sphere $S^{3}$ of radius $a$ and $h\left(D^{2} / \Lambda^{2}\right)$ is the Schwartz function in the spectral action, and $\beta$ is the size of the circle $S^{1}$, which has Dirac spectrum $\beta^{-1}(\mathbb{Z}+1 / 2)$.

One obtains then the spectral action on $S^{3} \times S^{1}$ using Poisson summation on $\mathbb{Z}^{2}$. This gives

$$
\operatorname{Tr}\left(h\left(D^{2} / \Lambda^{2}\right)\right)=\widehat{g}(0,0)+O\left(\Lambda^{-k}\right)
$$

for any $k>0$, where

$$
\widehat{g}(n, m)=\int_{\mathbb{R}^{2}} g(u, v) e^{-2 \pi i(x u+y v)} d u d v,
$$

and the error term $\sum_{(n, m) \neq(0,0)}(-1)^{n+m} \widehat{g}(n, m)$ is estimated to be smaller than $\Lambda^{-k}$. One obtains from (4.10)

$$
\operatorname{Tr}\left(h\left(D^{2} / \Lambda^{2}\right)\right)=\pi \Lambda^{4} a^{3} \beta \int_{0}^{\infty} u h(u) d u-\frac{1}{2} \pi \Lambda a \beta \int_{0}^{\infty} h(u) d u+O\left(\Lambda^{-k}\right) .
$$

One can consider particular classes of test functions $h$ which approximate well enough an even cutoff function on the Dirac spectrum. The class of functions used in [9] is test functions of the form $h(x)=P(\pi x) e^{-\pi x}$ with $P$ a polynomial, and in particular, among these, a good approximation to a cutoff function given by the test functions $h_{n}\left(x^{2}\right)$ with

$$
h_{n}(x)=\sum_{k=0}^{n} \frac{(\pi x)^{k}}{k !} e^{-\pi x} .
$$

\section{Slow-Roll potential From the SPeCtral aCtion}

We show here that the perturbations of the Dirac operator considered in [9], of the form $D^{2} \mapsto D^{2}+\phi^{2}$, give rise to a slow-roll potential for inflation. We compute the corresponding slow-roll parameters in the case of $S^{3}$. We then compute the potential and slow-roll parameters in the case of the other candidate cosmic topologies and we compare them with the case of $S^{3}$. 
5.1. Nonperturbative corrections and slow-roll potential. One of the most interesting aspects of the results of [9] is that, under the perturbation $D^{2} \mapsto D^{2}+\phi^{2}$ of the Dirac operator, one finds a potential $V(\phi)$ for a scalar field $\phi$ conformally coupled to gravity, which at low energies behaves like a quartic Higgs potential, but which has additional nonperturbative corrections, which have the effect, at higher energies of flattening out the form of the potential so that it is asymptotic to a constant. This gives it the typical form of the slow-roll potentials used in models of inflation in cosmology.

The replacement $D^{2} \mapsto D^{2}+\phi^{2}$, corresponding to a shift $h(u) \mapsto h(u+$ $\phi^{2} / \Lambda^{2}$ ) in the test function (assumed of the form $h(x)=P(\pi x) e^{-\pi x}$ as above) produces a potential for the field $\phi$, which, for sufficiently small values of the parameter $x=\phi^{2} / \Lambda^{2}$, recovers the usual quartic potential for the field $\phi$, conformally coupled to gravity, which on $S^{3} \times S^{1}$ is of the form

$$
-\pi \Lambda^{2} \beta a^{3} \int_{0}^{\infty} h(v) d v \phi^{2}+\frac{1}{2} \pi \beta a h(0) \phi^{2}+\frac{1}{2} \pi \beta a^{3} h(0) \phi^{4} .
$$

These correspond to a term of the form $\int_{X} R \phi^{2} d v o l$, giving the conformal coupling to gravity, from the 4th Seeley-de Witt coefficient, together with a quadratic mass term and a quartic potential, respectively from the second and 4 th coefficient.

However, for larger values of the parameter $x=\phi^{2} / \Lambda^{2}$, the potential obtained from the nonperturbative calculation of [9] levels out. Theorem 7 of [9] shows that one has on $S^{3} \times S^{1}$

$$
\begin{gathered}
\left.\operatorname{Tr}\left(h\left(\left(D^{2}+\phi^{2}\right) / \Lambda^{2}\right)\right)\right)=2 \pi \Lambda^{4} \beta a^{3} \int_{0}^{\infty} h\left(\rho^{2}\right) \rho^{3} d \rho-\pi \Lambda^{2} \beta a \int_{0}^{\infty} h\left(\rho^{2}\right) \rho d \rho \\
+\pi \Lambda^{4} \beta a^{3} \mathcal{V}\left(\phi^{2} / \Lambda^{2}\right)+\frac{\pi}{2} \Lambda^{2} \beta a \mathcal{W}\left(\phi^{2} / \Lambda^{2}\right)+\epsilon(\Lambda),
\end{gathered}
$$

where the error term $\epsilon(\Lambda)$ is exponentially small in $\Lambda$ and the funcions $\mathcal{V}$ and $\mathcal{W}$ are given by

$$
\mathcal{V}(x)=\int_{0}^{\infty} u(h(u+x)-h(u)) d u, \quad \mathcal{W}(x)=\int_{0}^{x} h(u) d u .
$$

This is the typical behavior expected from a slow-roll potential used in scenarios for inflationary cosmology based on the Standard Model of elementary particles, as in the recent paper [15. In particular, we are interested in deriving the associated slow-roll parameters.

5.2. Slow-roll parameters. A way to obtain models of inflation with slow roll potential is to have a theory with a non-minimal coupling of a scalar field to gravity via the curvature $R$. For a version of a Higgs based inflation see 15. We show here that the nonperturbative corrections to the Higgs potential in the spectral action obtained in [9] present a similar scenario. 
Notice that, in the derivation of the slow-roll potential from the spectral action, we have replaced the Minkowskian spacetime geometry with a compactified Euclidean model in order to compute the spectral action nonperturbatively and then derive the slow-roll potential from the perturbation of the Dirac operator. However, once we have obtained a Lagrangian for gravity coupled to a scalar field $\phi$ that will be responsible for inflation, we can continue the same Lagrangian back to Minkowskian signature and consider the effects of the slow-roll potential over a Minkowskian spacetime given by a Friedmann metric with assigned topology on the spatial sections.

Consider a Minkowskian space-time metric of the form

$$
d s^{2}=a(t)^{2} d s_{S}^{2}-d t^{2},
$$

where $d s_{S}^{2}$ is the assigned Riemannian homogeneous metric on the 3-manifold $S$ (the candidate cosmic topology) and $a(t)$ is the scale factor.

In models of inflations based on a scalar field with a slow-roll potential $V(\phi)$, the accelerated expansion phase $\ddot{a} / a>0$ is governed by the equation

$$
\frac{\ddot{a}}{a}=H^{2}(1-\epsilon),
$$

where the Hubble parameter $H^{2}(\phi)$ is related to the slow roll potential $V(\phi)$ by

$$
H^{2}(\phi)\left(1-\frac{1}{3} \epsilon(\phi)\right)=\frac{8 \pi}{3 m_{P l}^{2}} V(\phi),
$$

where $m_{P l}$ is the Planck mass and $\epsilon(\phi)$ is the first slow-roll parameter satisfying the equation of state

$$
\epsilon(\phi)=\frac{m_{P l}^{2}}{16 \pi}\left(\frac{V^{\prime}(\phi)}{V(\phi)}\right)^{2} .
$$

The inflationary phase is characterized by $\epsilon(\phi)<1$. The second slow-roll parameter has the form

$$
\eta(\phi)=\frac{m_{P l}^{2}}{8 \pi}\left(\frac{V^{\prime \prime}(\phi)}{V(\phi)}\right)-\frac{m_{P l}^{2}}{16 \pi}\left(\frac{V^{\prime}(\phi)}{V(\phi)}\right)^{2} .
$$

These parameters enter in two important measurable quantities: the spectral index and the tensor-to-scalar ratio, which are given respectively by

$$
\begin{aligned}
& n_{s}=1-6 \epsilon+2 \eta \\
& r=16 \epsilon .
\end{aligned}
$$

We remark that, from the cosmological viewpoint, the model we consider here will only be a toy model, in the sense that, as in [9] we only look at the purely gravitational part of the spectral action, and we do not consider the effect of the presence of matter coming from the presence of the additional noncommutative space as extra dimensions. This simplification has the advantage that it allows us to focus only on the nonperturbative effects on the Higgs potential, without having to carry around additional terms that 
are not directly affected by the 3-dimensional spatial topology. However, one should keep in mind that the resulting slow-roll parameters will also be affected by the matter contributions, as described in $\S 4$ of [25]. So, in particular, the values we obtain here for these parameters, in the simplifying assumption that drops the matter part, need not meet the observational constraints. The main point for us is to show that there is a contribution to these slow-roll parameters that can be different from that of the sphere in certain candidate cosmic topologies such as the flat tori or equal to that of the sphere in other candidate topologies such as quaternionic or dodecahedral spaces. For this reason we drop the matter terms that would not differ in the various cases.

5.3. Slow-roll parameters for the $S^{3}$-topology. We now compute the slow-roll parameters resulting from the nonperturbative corrections to the Higgs potential of [9], in the case where the underlying spatial topology is the 3 -sphere.

Theorem 5.1. The slow-roll potential

$$
V(x)=\pi \Lambda^{4} \beta a^{3} \mathcal{V}\left(\phi^{2} / \Lambda^{2}\right)+\frac{\pi}{2} \Lambda^{2} \beta a \mathcal{W}\left(\phi^{2} / \Lambda^{2}\right),
$$

with $\mathcal{V}$ and $\mathcal{W}$ as in (5.3), and $x=\phi^{2} / \Lambda^{2}$, has slow-roll parameters

$$
\epsilon(x)=\frac{m_{P l}^{2}}{16 \pi}\left(\frac{h(x)-2(\Lambda a)^{2} \int_{x}^{\infty} h(u) d u}{\int_{0}^{x} h(u) d u+2(\Lambda a)^{2} \int_{0}^{\infty} u(h(u+x)-h(u)) d u}\right)^{2}
$$

and

$$
\begin{aligned}
\eta(x) & =\frac{m_{P l}^{2}}{8 \pi} \frac{h^{\prime}(x)+2(\Lambda a)^{2} h(x)}{\int_{0}^{x} h(u) d u+2(\Lambda a)^{2} \int_{0}^{\infty} u(h(u+x)-h(u)) d u} \\
& -\frac{m_{P l}^{2}}{16 \pi}\left(\frac{h(x)-2(\Lambda a)^{2} \int_{x}^{\infty} h(u) d u}{\int_{0}^{x} h(u) d u+2(\Lambda a)^{2} \int_{0}^{\infty} u(h(u+x)-h(u)) d u}\right)^{2}
\end{aligned}
$$

written in the variable $x=\phi^{2} / \Lambda^{2}$.

Proof. We have, as in Lemma 8 of [9],

$$
\mathcal{V}^{\prime}(x)=-\int_{x}^{\infty} h(u) d u \quad \text { and } \quad \mathcal{V}^{\prime \prime}(x)=h(x),
$$

while $\mathcal{W}^{\prime}(x)=h(x)$ and $\mathcal{W}^{\prime \prime}(x)=h^{\prime}(x)$. So, if we write

$$
A=\frac{1}{2}\left(\frac{V^{\prime}(\phi)}{V(\phi)}\right)^{2} \quad \text { and } \quad B=\left(\frac{V^{\prime \prime}(\phi)}{V(\phi)}\right)
$$

so that

$$
\epsilon=\frac{m_{P l}^{2}}{8 \pi} A \quad \text { and } \quad \eta=\frac{m_{P l}^{2}}{8 \pi}(B-A)
$$


we find

$$
A=\frac{1}{2}\left(\frac{h(x)-2(\Lambda a)^{2} \int_{x}^{\infty} h(u) d u}{\int_{0}^{x} h(u) d u+2(\Lambda a)^{2} \int_{0}^{\infty} u(h(u+x)-h(u)) d u}\right)^{2}
$$

and

$$
B=\frac{h^{\prime}(x)+2(\Lambda a)^{2} h(x)}{\int_{0}^{x} h(u) d u+2(\Lambda a)^{2} \int_{0}^{\infty} u(h(u+x)-h(u)) d u}
$$

Remark 5.2. The slow-roll parameters obtained in this way are independent of the scale $\beta$, as one should expect since that was an artifact introduced by our passing to a Euclidean model to perform calculations with the spectral action, while they depend on both the energy scale $\Lambda$ and the scale factor $a$, but only through their product $\Lambda a$. This again fits in well with cosmological models, since we know that, for a cosmology described by a Friedmann metric (5.4), the time dependence of the energy scale factor is related through $\Lambda(t) \sim 1 / a(t)$, so that their product is a constant $C$ independent of time.

Thus, we can rewrite (5.9) and (5.10) for the 3 -sphere in the form

$$
\begin{aligned}
\epsilon(x) & =\frac{m_{P l}^{2}}{16 \pi}\left(\frac{h(x)-2 C \int_{x}^{\infty} h(u) d u}{\int_{0}^{x} h(u) d u+2 C \int_{0}^{\infty} u(h(u+x)-h(u)) d u}\right)^{2} \\
\eta(x) & =\frac{m_{P l}^{2}}{8 \pi} \frac{h^{\prime}(x)+2 \pi C h(x)}{\int_{0}^{x} h(u) d u+2 C \int_{0}^{\infty} u(h(u+x)-h(u)) d u} \\
& -\frac{m_{P l}^{2}}{16 \pi}\left(\frac{h(x)-2 C \int_{x}^{\infty} h(u) d u}{\int_{0}^{x} h(u) d u+2 \pi C \int_{0}^{\infty} u(h(u+x)-h(u)) d u}\right)^{2} .
\end{aligned}
$$

We now compare this inflation model derived from the nonperturbative spectral action on the sphere with the case of other nontrivial topologies.

\section{The quaternionic Cosmology and the SPeCtral aCtion}

Let $Q 8$ denote the group of quaternion units $\{ \pm 1, \pm i, \pm j, \pm k\}$. It acts on the 3 -sphere, with the latter identified with the group $S U(2)$. The resulting quotient manifold $S U(2) / Q 8$ plays an interesting role as a possible cosmic topology candidate, in view of the recent results of [28] on the statistical comparison of various spherical space forms in terms of the best fit for either the power spectrum $C_{\ell}$ or the off-diagonal part of the correlation matrices.

As shown in the study of correlation matrices, as exhibited in Figure 3 of 28, the quaternionic space, unlike the other nontrivial topologies considered in their study, shows no additional structure in the off-diagonal correlations with respect to the spherical case. Thus, the analysis of off-diagonal terms in the correlation functions does not suppress the Bayesian factor of the quaternionic space, while it suppresses those of all the other nontrivial topologies. While the other model comparison carried out in 28] using the 
power spectrum $C_{\ell}$ does not favor this topology, the particular behavior of the off-diagonal terms seems sufficiently interesting to develop additional possible tests for comparing the quaternionic geometry $S U(2) / Q 8$ to the ordinary spherical geometry.

6.1. The Dirac spectra for $S U(2) / Q 8$. As we show here, the main reason why the case of $S U(2) / Q 8$ can be treated with the same technique used in [9] for the sphere $S^{3}$ is because the Dirac spectrum is given in terms of arithmetic progressions indexed over the integers, so that one can again apply the same type of Poisson summation formula. This is not immediately the case for other spherical geometries.

More precisely, we recall from [17] that one can endow the 3-manifold $S U(2) / Q 8$ with a 3-parameter family of homogeneous metrics, depending on the parameters $a_{i} \in \mathbb{R}^{*}, i=1,2,3$. The different possible spin structures $\epsilon_{j}$ on $S U(2) / Q 8$ correspond to the four group homomorphisms $Q 8 \rightarrow \mathbb{Z} / 2 \mathbb{Z}$ with $\epsilon_{0} \equiv 1$ and $\operatorname{Ker}\left(\epsilon_{j}\right)=\left\{ \pm 1, \pm \sigma_{j}\right\}$, with $\sigma_{j}$ the Pauli matrices. The Dirac operator for each of these spin structures and its spectrum are computed explicitly in [17]. The case we are interested in here is only the one where the metric has parameters $a_{1}=a_{2}=a_{3}=1$, for which $S U(2) / Q 8$ is a spherical space form. For this case the Dirac spectrum was also computed in [2].

In this case, see Corollary 3.2 of [17], the Dirac spectrum for $S U(2) / Q 8$ with the spherical metric $a_{1}=a_{2}=a_{3}=1$, is given in the case of the spin structure $\epsilon_{0}$ by

$$
\left\{\begin{array}{lll}
\frac{3}{2}+4 k & \text { with multiplicity } & 2(k+1)(2 k+1) \\
\frac{3}{2}+4 k+2 & \text { with multiplicity } & 4 k(k+1) \\
-\frac{3}{2}-4 k-1 & \text { with multiplicity } & 2 k(2 k+1) \\
-\frac{3}{2}-4 k-3 & \text { with multiplicity } & 4(k+1)(k+2)
\end{array}\right.
$$

where $k$ runs over $\mathbb{N}$. For all the other three spin structures $\epsilon_{j}, j=1,2,3$, the spectrum is given by

$$
\left\{\begin{array}{lll}
\frac{3}{2}+4 k & \text { with multiplicity } & 2 k(2 k+1) \\
\frac{3}{2}+4 k+2 & \text { with multiplicity } & 4(k+1)^{2} \\
-\frac{3}{2}-4 k-1 & \text { with multiplicity } & 2(k+1)(2 k+1) \\
-\frac{3}{2}-4 k-3 & \text { with multiplicity } & 4(k+1)^{2},
\end{array}\right.
$$

again with $k \in \mathbb{N}$.

6.2. Trivial spin structure: nonperturbative spectral action. By replacing $k$ with $-k-1$ in the third row and $k$ with $-k-2$ in the fourth row, 
we rewrite the spectrum (6.1) in the form

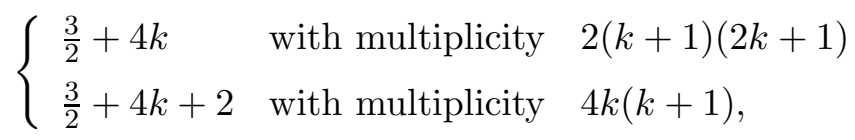

where now $k$ runs over the integers $\mathbb{Z}$. This expresses the spectrum in terms of two arithmetic progressions indexed over the integers. Now the condition that allows us to apply the Poisson summation formula as in 9] is the fact that the multiplicities can be expressed in terms of a smooth function of $k$. This is the case, since the multiplicites in (6.3) for an eigenvalue $\lambda$ are given, respectively, by the functions $P_{1}(\lambda)$ and $P_{2}(\lambda)$ with

$$
\begin{aligned}
& P_{1}(u)=\frac{1}{4} u^{2}+\frac{3}{4} u+\frac{5}{16} \\
& P_{2}(u)=\frac{1}{4} u^{2}-\frac{3}{4} u-\frac{7}{16} .
\end{aligned}
$$

We then obtain an explicit nonperturbative calculation of the spectral action for $S U(2) / Q 8$ as follows.

Theorem 6.1. The spectral action on the 3-manifold $S=S U(2) / Q 8$, with the trivial spin structure, is given by

$$
\operatorname{Tr}(f(D / \Lambda))=\frac{1}{8}(\Lambda a)^{3} \widehat{f}^{(2)}(0)-\frac{1}{32}(\Lambda a) \widehat{f}(0)+\epsilon(\Lambda),
$$

with a the radius of the 3-sphere $S U(2)=S^{3}$, with the error term satisfying $|\epsilon(\Lambda)|=O\left(\Lambda^{-k}\right)$ for all $k>0$, and with $\widehat{f}^{(k)}$ denoting the Fourier transform of $v^{k} f(v)$ as above. Namely, the spectral action for $S U(2) / Q 8$ is $1 / 8$ of the spectral action for $S^{3}$.

Proof. Consider a test function for the Poisson summation formula which is of the form

$$
h(u)=g\left(4 u+\frac{s}{2}\right), \quad \text { for some } \quad s \in \mathbb{Z} .
$$

Then (4.4) gives

$$
\sum_{n \in \mathbb{Z}} g\left(4 n+\frac{s}{2}\right)=\sum_{n \in \mathbb{Z}} \frac{1}{4} \exp \left(\frac{i \pi s n}{4}\right) \widehat{g}\left(\frac{n}{4}\right),
$$

which we apply to $g_{i}(u)=P_{i}(u) f(u / \Lambda)$, with $P_{i}$ as in (6.4) and $f$ the Schwartz function in the spectral action approximating a cutoff.

This gives an expression for the spectral action on $S=S U(2) / Q 8$ with the trivial spin structure, and with the sphere $S^{3}=S U(2)$ of radius one, which is of the form

$$
\begin{aligned}
\operatorname{Tr}(f(D / \Lambda)) & =\sum_{\mathbb{Z}} g_{1}\left(4 n+\frac{3}{2}\right)+\sum_{\mathbb{Z}} g_{2}\left(4 n+\frac{7}{2}\right) \\
& =\sum_{\mathbb{Z}} \frac{1}{4} \exp \left(\frac{3 \pi i n}{4}\right) \widehat{g}_{1}\left(\frac{n}{4}\right)+\sum_{\mathbb{Z}} \frac{1}{4} \exp \left(\frac{7 \pi i n}{4}\right) \widehat{g}_{2}\left(\frac{n}{4}\right) .
\end{aligned}
$$


Assuming that $f$ is a Schwartz function, then $g_{i}$ is also Schwartz, hence so is $\widehat{g}_{i}$. Therefore, for each $k \in \mathbb{N}$, we get an estimate of the form

$$
\sum_{n \neq 0} \frac{1}{4}\left|\widehat{g}_{i}\left(\frac{n}{4}\right)\right| \leq C_{k} \Lambda^{-k} .
$$

This shows that we can write the right hand side of (6.7) as the terms involving $\widehat{g}_{i}(0)$ plus an error term that is of order $O\left(\Lambda^{-k}\right)$.

One then computes

$$
\widehat{g}_{1}(0)=\frac{1}{4} \Lambda^{3} \widehat{f}^{(2)}(0)+\frac{3}{4} \Lambda^{2} \widehat{f}^{(1)}(0)+\frac{5}{16} \Lambda \widehat{f}(0) .
$$

Similarly, on has

$$
\widehat{g}_{2}(0)=\frac{1}{4} \Lambda^{3} \widehat{f}^{(2)}(0)-\frac{3}{4} \Lambda^{2} \widehat{f}^{(1)}(0)-\frac{7}{16} \Lambda \widehat{f}(0),
$$

so that one obtains for the spectral action in (6.7)

$$
\begin{aligned}
\operatorname{Tr}(f(D / \Lambda)) & =\frac{1}{4}\left(\widehat{g}_{1}(0)+\widehat{g}_{2}(0)\right)+O\left(\Lambda^{-k}\right) \\
& =\frac{1}{8} \Lambda^{3} \widehat{f}^{(2)}(0)-\frac{1}{32} \Lambda \widehat{f}(0)+O\left(\Lambda^{-k}\right) .
\end{aligned}
$$

The case with the 3 -sphere $S U(2)=S^{3}$ of radius $a$ is then analogous, with the spectrum scaled by a factor of $a^{-1}$, which is like changing $\Lambda$ to $\Lambda a$ in the expressions above, so that one obtains (6.5).

6.3. Nontrivial spin structures: nonperturbative spectral action. The computation of the spectral action on $S U(2) / Q 8$ in the case of the nontrivial spin structures $\epsilon_{j}$ with $j=1,2,3$ is analogous. One starts with the Dirac spectrum (6.2) and writes it in the form of two arithmetic progressions indexed over the integers

$$
\left\{\begin{array}{lll}
\frac{3}{2}+4 k & \text { with multiplicity } & 2 k(2 k+1) \\
\frac{3}{2}+4 k+2 & \text { with multiplicity } & 4(k+1)^{2}
\end{array}\right.
$$

In this case one again has polynomials interpolating the values of the multiplicities. They are of the form

$$
\begin{aligned}
& P_{1}(u)=\frac{1}{4} u^{2}-\frac{1}{4} u-\frac{3}{16} \\
& P_{2}(u)=\frac{1}{4} u^{2}+\frac{1}{4} u+\frac{1}{16} .
\end{aligned}
$$

We then obtain the following result.

Theorem 6.2. The spectral action on the 3-manifold $S=S U(2) / Q 8$, for any of the non-trivial spin structures $\epsilon_{j}, j=1,2,3$, is given by the same expression (6.5) as in the case of the trivial spin structure $\epsilon_{0}$. 
Proof. It is enough to observe that the sum of the two polynomials (6.12) that interpolate the spectral multiplicities,

$$
P_{1}(u)+P_{2}(u)=\frac{1}{2} u^{2}-\frac{1}{8}
$$

is the same as in the case (6.4) of the trivial spin structure. One then has the same value of

$$
\frac{1}{4} \widehat{g}_{1}(0)+\frac{1}{4} \widehat{g}_{2}(0)=\frac{1}{4} \int_{\mathbb{R}}\left(P_{1}(u)+P_{2}(u)\right) f(u / \Lambda) d u,
$$

which gives the spectral action up to an error term of the order of $O\left(\Lambda^{-k}\right)$.

\subsection{Slow-roll potential and parameters for the quaternionic space.}

We compute now the slow-roll potential and slow-roll parameters for the case of the quaternionic cosmic topology $S=S U(2) / Q 8$. We first compute the spectral action in the Euclidean 4-dimensional model $S \times S^{1}$, from which we obtain the slow-roll potential by a perturbation of the Dirac operator as in the case of $S^{3}$.

Theorem 6.3. The spectral action on the 4-manifold $S \times S^{1}$ with $S=$ $S U(2) / Q 8$ is given by

$$
\operatorname{Tr}\left(h\left(D^{2} / \Lambda^{2}\right)\right)=\frac{\pi}{8} \Lambda^{4} a^{3} \beta \int_{0}^{\infty} u h(u) d u-\frac{\pi}{16} \Lambda^{2} a \beta \int_{0}^{\infty} h(u) d u+O\left(\Lambda^{-k}\right),
$$

namely $1 / 8$ of the spectral action for $S^{3} \times S^{1}$.

Proof. The eigenvalues for the operator $D^{2} / \Lambda^{2}$ on $S \times S^{1}$ are

$$
\left(4 k+\frac{s}{2}\right)^{2}(\Lambda a)^{-2}+\left(m+\frac{1}{2}\right)^{2}(\Lambda \beta)^{-2},
$$

for $S U(2)=S^{3}$ of radius $a$ and $S^{1}$ of radius $\beta$, with multiplicities $2 P_{i}(u)$, where $P_{i}(u)$ are the polynomials (6.4) and (6.12) that interpolate the spectral densities for $S=S U(2) / Q 8$ and the integer $s$ also varies according to the arithmetic progressions in the spectrum (6.3) or (6.11).

For a given integer $s$, the Poisson summation formula over $\mathbb{Z}^{2}$ gives

$$
\sum_{(n, m) \in \mathbb{Z}^{2}} g\left(4 n+\frac{s}{2}, m+\frac{1}{2}\right)=\sum_{(n, m) \in \mathbb{Z}^{2}} \frac{1}{4} \exp \left(\frac{\pi i n s}{4}\right)(-1)^{m} \widehat{g}\left(\frac{n}{4}, m\right),
$$

where $g$ is a Schwartz function of the form (4.9). We have two functions

$$
g_{i}(u, v)=2 P_{i}(u) h\left(u^{2}(\Lambda a)^{-2}+v^{2}(\Lambda \beta)^{-2}\right),
$$

with $P_{i}$ as in (6.4) and (6.12), respectively, for the trivial and non-trivial spin structure. 
In the case of the trivial spin structure one writes the spectral action as (6.16)

$$
\begin{aligned}
\operatorname{Tr}\left(h\left(D^{2} / \Lambda^{2}\right)\right) & =\sum_{\mathbb{Z}^{2}} g_{1}\left(4 n+\frac{3}{2}, m+\frac{1}{2}\right)+\sum_{\mathbb{Z}^{2}} g_{2}\left(4 n+\frac{7}{2}, m+\frac{1}{2}\right) \\
& =\sum_{\mathbb{Z}^{2}} \frac{1}{4} \exp \left(\frac{3 \pi i n}{4}\right)(-1)^{m} \widehat{g}_{1}\left(\frac{n}{4}, m\right) \\
& +\sum_{\mathbb{Z}^{2}} \frac{1}{4} \exp \left(\frac{7 \pi i n}{4}\right)(-1)^{m} \widehat{g}_{2}\left(\frac{n}{4}, m\right) .
\end{aligned}
$$

The main term that contributes to (6.16) is

$$
\begin{aligned}
& \widehat{g}_{1}(0,0)+\widehat{g}_{2}(0,0) \\
& =2 \Lambda^{2} a \beta \int_{\mathbb{R}^{2}}\left(P_{1}(\Lambda a x)+P_{2}(\Lambda a x)\right) h\left(x^{2}+y^{2}\right) d x d y \\
& =\Lambda^{2} a \beta \int_{\mathbb{R}^{2}}\left((\Lambda a)^{2} x^{2}-\frac{1}{4}\right) h\left(x^{2}+y^{2}\right) d x d y \\
& =\pi \Lambda^{4} a^{3} \beta \int_{0}^{\infty} h\left(\rho^{2}\right) \rho^{3} d \rho-\frac{\pi}{2} \Lambda^{2} a \beta \int_{0}^{\infty} h\left(\rho^{2}\right) \rho d \rho .
\end{aligned}
$$

The error term

$$
\sum_{(n, m) \neq(0,0)} \frac{1}{4}\left|\widehat{g}_{i}\left(\frac{n}{4}, m\right)\right|
$$

can be estimated as in [9] and is of the order of $O\left(\Lambda^{-k}\right)$, for all $k>0$.

The result for the non-trivial spin structures is the same, since we have seen that the sum $P_{1}(u)+P_{2}(u)$ is the same. This gives (6.13) after the change of variables

$$
\int_{0}^{\infty} h\left(\rho^{2}\right) \rho^{3} d \rho=\frac{1}{2} \int_{0}^{\infty} u h(u) d u \quad \text { and } \quad \int_{0}^{\infty} h\left(\rho^{2}\right) \rho d \rho=\frac{1}{2} \int_{0}^{\infty} h(u) d u .
$$

Since the spectral action for $S \times S^{1}$ in this case only differs from the one of $S^{3} \times S^{1}$ by a the multiplicative factor $1 / 8$, we obtain the following for the slow-roll potential and the slow-roll parameters.

Proposition 6.4. The slow-roll potential for $S=S U(2) / Q 8$ is

$$
V(\phi)=\frac{1}{8} \pi \Lambda^{4} \beta a^{3} \mathcal{V}\left(\phi^{2} / \Lambda^{2}\right)+\frac{1}{16} \pi \Lambda^{2} \beta a \mathcal{W}\left(\phi^{2} / \Lambda^{2}\right),
$$

with $\mathcal{V}$ and $\mathcal{W}$ as in (5.3), and the slow-roll parameters are the same (5.11) as for the 3-sphere.

Thus, in this case the slow-roll inflation model derived from the spectral action does not distinguish the standard cosmic topology $S^{3}$ from the quaternionic case $S U(2) / Q 8$. 


\section{Poincaré homology SPhere: Dodecahedral COSMOlogy}

The Poincare homology sphere, which is the quotient of the 3 -sphere $S^{3}$ by the binary icosahedral group $\Gamma$, is also commonly referred to as the dodecahedral space, due to the fact that the action of $\Gamma$ on $S^{3}$ has a fundamental domain that is a dodecahedron. The dodecahedral space is obtained by gluing together opposite faces of a dodecahedron with the shortest clockwise twist that matches the faces. This space has been regarded as a likely candidate for the cosmic topology problem and extensively studied for testable cosmological signatures with all the methods presently available, 7], [16], [22], 24], 28], 31, 333, 39], 40].

In particular, the three-year WMAP results confirmed the main anomalies: quadrupole suppression, small value of the two-point temperature correlation function at large angles, and quadrupole-octupole alignment. A recent analysis [7] of the Poincaré dodecahedral space based on the explicit computation of the Laplace spectrum and the construction of the resulting simulated CMB sky with more precise estimates of higher modes up to $\ell \sim 30$ finds a good match to the WMAP data regarding the two-point temperature correlation function. Thus, the dodecahedral space remains at present one of the most likely candidates, although it fails to account for other anomalies like the quadrupole-octupole alignment 39 .

We give here the explicit computation of the spectral action functional for the dodecahedral space, and we show then in $\$ 7.6$ that, in our model, from the point of view of the resulting inflation slow-roll parameters, the dodecahedral space behaves like the sphere, so that it cannot be ruled out as a candidate cosmic topology in a gravity model based on the spectral action.

7.1. Generating functions for spectral multiplicities. To compute explicitly the Dirac spectrum of the Poincaré homology sphere, we use a general result of Bär [2], which gives a formula for the generating function of the spectral multiplicities of the Dirac spectrum on space forms of positive curvature.

In the generality of [2], one considers a manifold $M$ that is a quotient $M=S^{n} / \Gamma$ of an $n$-dimensional sphere, $n \geq 2$, with the standard metric of curvature one, with $\Gamma \subset S O(n+1)$ a finite group acting without fixed points. It is shown in [2] that the classical Dirac operator on $S^{n}$ has spectrum

$$
\pm\left(\frac{n}{2}+k\right), k \geq 0, \quad \text { with multiplicities } \quad 2^{[n / 2]}\left(\begin{array}{c}
k+n-1 \\
k
\end{array}\right) .
$$

The eigenvalues of $M$ are the same as the eigenvalues of $S^{n}$, but with smaller multiplicities. The spin structures of $M$ are in 1-1 corrrespondence with homomorphisms $\epsilon: \Gamma \rightarrow \operatorname{Spin}(n+1)$, such that $\Theta \circ \epsilon=i d_{\Gamma}$, where $\Theta$ is simply the double cover map from $\operatorname{Spin}(n+1)$ to $S O(n+1)$. If $D$ is the Dirac operator on $\mathrm{M}$, then to specify the spectrum of $M$, for one of these spin structures, one just needs to know the multiplicities, $m( \pm(n / 2+k))$, 
$k \geq 0$. These are encoded in two generating functions

$$
\begin{aligned}
& F_{+}(z)=\sum_{k=0}^{\infty} m\left(\frac{n}{2}+k, D\right) z^{k} \\
& F_{-}(z)=\sum_{k=0}^{\infty} m\left(-\left(\frac{n}{2}+k\right), D\right) z^{k} .
\end{aligned}
$$

It is elementary to show that these power series have radius of convergence at least 1 about $z=0$.

Now denote the irreducible half spin representations of $\operatorname{Spin}(2 m)$ by

$$
\begin{aligned}
& \rho^{+}: \operatorname{Spin}(2 m) \rightarrow \operatorname{Aut}\left(\Sigma_{2 m}^{+}\right) \\
& \rho^{-}: \operatorname{Spin}(2 m) \rightarrow \operatorname{Aut}\left(\Sigma_{2 m}^{-}\right),
\end{aligned}
$$

where $\Sigma_{2 m}^{ \pm}$are the positive and negative spinor spaces. Let $\chi^{ \pm}: \operatorname{Spin}(2 m) \rightarrow$ $\mathbb{C}$ be the character of $\rho^{ \pm}$. It is shown in [2] that the generating functions of the spectral multiplicities have the form

$$
\begin{aligned}
& F_{+}(z)=\frac{1}{|\Gamma|} \sum_{\gamma \in \Gamma} \frac{\chi^{-}(\epsilon(\gamma))-z \cdot \chi^{+}(\epsilon(\gamma))}{\operatorname{det}\left(1_{2 m}-z \cdot \gamma\right)} \\
& F_{-}(z)=\frac{1}{|\Gamma|} \sum_{\gamma \in \Gamma} \frac{\chi^{+}(\epsilon(\gamma))-z \cdot \chi^{-}(\epsilon(\gamma))}{\operatorname{det}\left(1_{2 m}-z \cdot \gamma\right)}
\end{aligned}
$$

7.2. The Dirac spectrum of the Poincaré sphere. In order to compute explicitly the Dirac spectrum of the Poincaré homology sphere, it suffices then to compute the multiplicities by computing explicitly the generating functions (7.4) and (7.5).

Let $\Gamma$ be the binary icosahedral group. To carry out our computations, we regard $S^{3}$ as being the set of unit quaternions, and $\Gamma$ is the following set of 120 unit quaternions:

- 24 elements are as follows, where the signs in the last group are chosen independently of one another:

$$
\left\{ \pm 1, \pm i, \pm j, \pm k, \frac{1}{2}( \pm 1 \pm i \pm j \pm k)\right\} .
$$

- 96 elements are either of the following form, or obtained by an even permutation of coordinates of the following form:

$$
1 / 2\left(0 \pm i \pm \phi^{-1} j \pm \phi k\right)
$$

where $\phi$ is the golden ratio.

Then $\Gamma$ acts on $S^{3}$ by left multiplication. Similarly, if $S^{3}$ is regarded as the unit sphere in $\mathbb{R}^{4}$, then $S O(4)$ acts on $S^{3}$ by left multiplication. In this way, 
we may identify $a+b i+c j+d k \in \Gamma$, with the following matrix in $S O(4)$ :

$$
\left(\begin{array}{cccc}
a & -b & -c & -d \\
b & a & -d & c \\
c & d & a & -b \\
d & -c & b & a
\end{array}\right)
$$

7.3. The double cover $\operatorname{Spin}(4) \rightarrow S O(4)$. Let us recall some facts about the double cover $\operatorname{Spin}(4) \rightarrow S O(4)$. Let $S_{L}^{3} \simeq S U(2)$ be the group of left isoclinic rotations:

$$
\left(\begin{array}{cccc}
a & -b & -c & -d \\
b & a & -d & c \\
c & d & a & -b \\
d & -c & b & a
\end{array}\right)
$$

where $a^{2}+b^{2}+c^{2}+d^{2}=1$. Similarly, let $S_{R}^{3} \simeq S U(2)$ be the group of right isoclinic rotations:

$$
\left(\begin{array}{cccc}
p & -q & -r & -s \\
q & p & s & -r \\
r & -s & p & q \\
s & r & -q & p
\end{array}\right)
$$

where $p^{2}+q^{2}+r^{2}+s^{2}=1$. Then $\operatorname{Spin}(4) \simeq S_{L}^{3} \times S_{R}^{3}$, and the double cover $\Theta: \operatorname{Spin}(4) \rightarrow S O(4)$ is given by $(A, B) \mapsto A \cdot B$, where $A \in S_{L}^{3}$, and $B \in S_{R}^{3}$. The complex half-spin representation $\rho^{-}$is just the projection onto $S_{L}^{3}$, where we identify $S_{L}^{3}$ with $S U(2)$ via

$$
\left(\begin{array}{cccc}
a & -b & -c & -d \\
b & a & -d & c \\
c & d & a & -b \\
d & -c & b & a
\end{array}\right) \mapsto\left(\begin{array}{cc}
a-b i & d+c i \\
-d+c i & a+b i
\end{array}\right)
$$

The other complex half-spin representation $\rho^{+}$is the projection onto $S_{R}^{3}$, where we identify $S_{R}^{3}$ with $S U(2)$ via

$$
\left(\begin{array}{cccc}
p & -q & -r & -s \\
q & p & s & -r \\
r & -s & p & q \\
s & r & -q & p
\end{array}\right)^{t} \mapsto\left(\begin{array}{cc}
p-q i & s+r i \\
-s+r i & p+q i
\end{array}\right)
$$

7.4. The spectral multiplicities. We define our spin structure $\epsilon: \Gamma \rightarrow$ $\operatorname{Spin}(4)$ to simply be $A \mapsto\left(A, I_{4}\right)$. It is obvious that this map satisfies $\Theta \circ \epsilon=i d_{\Gamma}$. Therefore, given $\gamma=a+b i+c j+d k \in \Gamma$, we see that

$$
\begin{aligned}
& \chi^{-}(\epsilon(\gamma))=2 a \\
& \chi^{+}(\epsilon(\gamma))=2 .
\end{aligned}
$$

We then obtain the following result by direct computation of the expressions (7.4) and (7.5), substituting the explicit expressions for all the group elements. This can be done using Mathematica. 
Theorem 7.1. Let $S=S^{3} / \Gamma$ be the Poincaré sphere, with the spin structure $\epsilon$ described here above. The generating functions for the spectral multiplicities of the Dirac operator are

$$
F_{+}(z)=-\frac{16(710647+317811 \sqrt{5}) G^{+}(z)}{(7+3 \sqrt{5})^{3}(2207+987 \sqrt{5}) H^{+}(z)}
$$

where

$$
\begin{aligned}
G^{+}(z) & =6 z^{11}+18 z^{13}+24 z^{15}+12 z^{17}-2 z^{19} \\
& -6 z^{21}-2 z^{23}+2 z^{25}+4 z^{27}+3 z^{29}+z^{31}
\end{aligned}
$$

and

$$
\begin{aligned}
H^{+}(z) & =-1-3 z^{2}-4 z^{4}-2 z^{6}+2 z^{8}+6 z^{10}+9 z^{12}+9 z^{14}+4 z^{16} \\
& -4 z^{18}-9 z^{20}-9 z^{22}-6 z^{24}-2 z^{26}+2 z^{28}+4 z^{30}+3 z^{32}+z^{34}
\end{aligned}
$$

and

$$
F_{-}(z)=-\frac{1024(5374978561+2403763488 \sqrt{5}) G^{-}(z)}{(7+3 \sqrt{5})^{8}(2207+987 \sqrt{5}) H^{-}(z)}
$$

where

$$
\begin{aligned}
G^{-}(z) & =1+3 z^{2}+4 z^{4}+2 z^{6}-2 z^{8}-6 z^{10} \\
& -2 z^{12}+12 z^{14}+24 z^{16}+18 z^{18}+6 z^{20}
\end{aligned}
$$

and

$$
\begin{aligned}
H^{-}(z) & =-1-3 z^{2}-4 z^{4}-2 z^{6}+2 z^{8}+6 z^{10}+9 z^{12}+9 z^{14}+4 z^{16} \\
& -4 z^{18}-9 z^{20}-9 z^{22}-6 z^{24}-2 z^{26}+2 z^{28}+4 z^{30}+3 z^{32}+z^{34} .
\end{aligned}
$$

We can then obtain explicitly the spectral multiplicities from the Taylor coefficients of $F_{+}(z)$ and $F_{-}(z)$, as in 7.2 and 7.3 ,

7.5. The spectral action for the Poincaré sphere. In order to compute the spectral action, we proceed as in the previous cases by identifying polynomials whose values at the points of the spectrum give the values of the spectral multiplicities. We obtain the following result.

Proposition 7.2. There are polynomials $P_{k}(u)$, for $k=0, \ldots, 59$, so that $P_{k}(3 / 2+k+60 j)=m(3 / 2+k+60 j, D)$ for all $j \in \mathbb{Z}$. The $P_{k}(u)$ are given 
as follows:

$$
\begin{aligned}
P_{k} & =0, \quad \text { whenever } k \text { is even. } \\
P_{1}(u) & =\frac{1}{48}-\frac{1}{20} u+\frac{1}{60} u^{2} . \\
P_{3}(u) & =\frac{3}{80}-\frac{1}{12} u+\frac{1}{60} u^{2} . \\
P_{5}(u) & =\frac{13}{240}-\frac{7}{60} u+\frac{1}{60} u^{2} . \\
P_{7}(u) & =\frac{17}{240}-\frac{3}{20} u+\frac{1}{60} u^{2} . \\
P_{9}(u) & =\frac{7}{80}-\frac{11}{60} u+\frac{1}{60} u^{2} .
\end{aligned}
$$

$$
\begin{aligned}
& P_{11}(u)=-\frac{19}{48}+\frac{47}{60} u+\frac{1}{60} u^{2} . \\
& P_{13}(u)=\frac{29}{240}-\frac{1}{4} u+\frac{1}{60} u^{2} . \\
& P_{15}(u)=\frac{11}{80}-\frac{17}{60} u+\frac{1}{60} u^{2} . \\
& P_{17}(u)=\frac{37}{240}-\frac{19}{60} u+\frac{1}{60} u^{2} . \\
& P_{19}(u)=-\frac{79}{240}+\frac{13}{20} u+\frac{1}{60} u^{2} .
\end{aligned}
$$

$$
\begin{aligned}
& P_{21}(u)=\frac{3}{16}-\frac{23}{60} u+\frac{1}{60} u^{2} . \\
& P_{23}(u)=-\frac{71}{240}+\frac{7}{12} u+\frac{1}{60} u^{2} . \\
& P_{25}(u)=\frac{53}{240}-\frac{9}{20} u+\frac{1}{60} u^{2} . \\
& P_{27}(u)=\frac{19}{80}-\frac{29}{60} u+\frac{1}{60} u^{2} . \\
& P_{29}(u)=-\frac{59}{240}+\frac{29}{60} u+\frac{1}{60} u^{2} .
\end{aligned}
$$




$$
\begin{aligned}
& P_{31}(u)=-\frac{11}{48}+\frac{9}{20} u+\frac{1}{60} u^{2} . \\
& P_{33}(u)=\frac{23}{80}-\frac{7}{12} u+\frac{1}{60} u^{2} . \\
& P_{35}(u)=-\frac{47}{240}+\frac{23}{60} u+\frac{1}{60} u^{2} . \\
& P_{37}(u)=\frac{77}{240}-\frac{13}{20} u+\frac{1}{60} u^{2} . \\
& P_{39}(u)=-\frac{13}{80}+\frac{19}{60} u+\frac{1}{60} u^{2} . \\
& P_{41}(u)=-\frac{7}{48}+\frac{17}{60} u+\frac{1}{60} u^{2} . \\
& P_{43}(u)=-\frac{31}{240}+\frac{1}{4} u+\frac{1}{60} u^{2} . \\
& P_{45}(u)=\frac{31}{80}-\frac{47}{60} u+\frac{1}{60} u^{2} . \\
& P_{47}(u)=-\frac{23}{240}+\frac{11}{60} u+\frac{1}{60} u^{2} . \\
& P_{49}(u)=-\frac{19}{240}+\frac{3}{20} u+\frac{1}{60} u^{2} . \\
& P_{57}(u)=\frac{39}{80}-\frac{59}{60} u+\frac{1}{60} u^{2} . \\
& P_{59}(u)=-\frac{119}{240}+\frac{59}{60} u+\frac{1}{60} u^{2} . \\
& P_{51}(u)=-\frac{1}{16}+\frac{7}{60} u+\frac{1}{60} u^{2} . \\
& P_{53}(u)=-\frac{11}{240}+\frac{1}{12} u+\frac{1}{60} u^{2} . \\
& P_{55}(u)=-\frac{1}{240}+\frac{1}{20} u+\frac{1}{60} u^{2} . \\
& P^{2} .
\end{aligned}
$$

Proof. These are computed directly from the Taylor coefficients of the generating functions of the spectral multiplicities (7.8) and (7.9).

We then obtain the nonperturbative spectral action for the Poincaré sphere.

Theorem 7.3. Let $D$ be the Dirac operator on the Poincaré homology sphere $S=S^{3} / \Gamma$, with the spin structure $\epsilon: \Gamma \rightarrow \operatorname{Spin}(4)$ with $A \mapsto\left(A, I_{4}\right)$. Then, for $f$ a Schwartz function, the spectral action is given by

$$
\operatorname{Tr}(f(D / \Lambda))=\frac{1}{60}\left(\frac{1}{2} \Lambda^{3} \widehat{f}^{(2)}(0)-\frac{1}{8} \Lambda \widehat{f}(0)\right),
$$


which is precisely 1/120 of the spectral action on the sphere.

Proof. The result follows by applying Poisson summation again, to the functions $g_{j}(u)=P_{j}(u) f(u / \Lambda)$. This gives, up to an error term which is of the order of $O\left(\Lambda^{-k}\right)$ for any $k>0$, the spectral action in the form

$$
\operatorname{Tr}(f(D / \Lambda))=\frac{1}{60} \sum_{j=0}^{59} \widehat{g}_{j}(0)=\frac{1}{60} \int_{\mathbb{R}} \sum_{j} P_{j}(u) f(u / \Lambda) d u .
$$

It suffices then to notice that

$$
\sum_{j=0}^{59} P_{j}(u)=\frac{1}{2} u^{2}-\frac{1}{8}
$$

The result then follows as in the sphere case.

7.6. Slow-roll potential in dodecahedral cosmologies. The dodecahedral space $S=S^{3} / \Gamma$, with $\Gamma$ the binary icosahedral group, also behaves in the same way as the quaternionic space $S U(2) / Q 8$ with respect to the properties of the slow-roll potential and slow-roll parameters. Namely, the slow-roll potential is a multiple of the potential for the sphere $S^{3}$ and the slow-roll parameters are therefore equal to those of the sphere.

Theorem 7.4. The spectral action for the manifold $S \times S^{1}$, with $S=S^{3} / \Gamma$ the Poincaré dodecahedral space, is given by 1/120 of the spectral action of $S^{3} \times S^{1}$ (4.12),

$$
\operatorname{Tr}\left(h\left(D^{2} / \Lambda^{2}\right)\right) \sim \frac{\pi}{120} \Lambda^{4} a^{3} \beta \int_{0}^{\infty} u h(u) d u-\frac{\pi}{240} \Lambda^{2} a \beta \int_{0}^{\infty} h(u) d u,
$$

up to an error term of the order of $O\left(\Lambda^{-k}\right)$. The slow-roll potential $V(\phi)$ obtained by replacing $D^{2} \mapsto D^{2}+\phi^{2}$ is also $1 / 120$ of the potential for the 3-sphere,

$$
V(\phi)=\frac{\pi}{120} \Lambda^{4} \beta a^{3} \mathcal{V}\left(\phi^{2} / \Lambda^{2}\right)+\frac{\pi}{240} \Lambda^{2} \beta a \mathcal{W}\left(\phi^{2} / \Lambda^{2}\right),
$$

with $\mathcal{V}$ and $\mathcal{W}$ as in (5.3). The slow-roll parameters are the same (5.9), (5.10) as for the sphere $S^{3}$.

Proof. We showed in Theorem 7.3 that the spectral action for the Poincaré dodecahedral space $S=S^{3} / \Gamma$ is $1 / 120$ of the spectral action of the 3 -sphere of radius one. Changing the radius $a$ of the 3 -sphere has the effect of changing $\Lambda \mapsto(\Lambda a)$ in the expression (7.10) of the spectral action, as in the case of the sphere. We then obtain the spectral action $\operatorname{Tr}\left(h\left(D^{2} / \Lambda^{2}\right)\right)$ for the product $S \times S^{1}$, as in Theorem 6.3 using Poisson summation applied to the functions

$$
g_{i}(u, v)=2 P_{i}(u) h\left(u^{2}(\Lambda a)^{-2}+v^{2}(\Lambda \beta)^{-2}\right),
$$


with $P_{i}(u)$ as in Proposition 7.2, namely

$$
\operatorname{Tr}\left(h\left(D^{2} / \Lambda^{2}\right)\right)=\sum_{(n, m) \in \mathbb{Z}^{2}} \sum_{i=0}^{59} g_{i}\left(60 n+i+\frac{3}{2}, m+\frac{1}{2}\right) .
$$

Then, the Poisson summation formula applied to these functions shows that the spectral action on the product of the dodecahedral space by the circle is given by

$$
\operatorname{Tr}\left(h\left(D^{2} / \Lambda^{2}\right)\right)=\frac{1}{60} \sum_{i} \widehat{g}_{i}(0,0)+O\left(\Lambda^{-k}\right) .
$$

We compute this as in the sphere case, using the fact that $\sum_{i} P_{i}(u)=u^{2} / 2-$ $1 / 8$. This gives (7.11), as in the case of the sphere and of the quaternionic space. The slow-roll potential is then obtained exactly as in the previous cases.

\section{Flat cosmologies}

Another very promising candidate for possible non-simply-connected cosmic topologies is given by the flat manifolds: flat 3-dimensional tori and their quotients, the Bieberbach manifolds.

Simulated CMB skies have been computed for tori and for all the Bieberbach manifolds in [32. The method is the same as in the analysis of simulated CMB skies for spherical space forms of [23], [31, namely through the explicit computation of the spectrum and eigenforms of the Laplacian. In the case of flat tori, the basis given by planar waves is more directly adapted to the topology, while the basis in spherical waves is better suited for comparison between simulated and observed CMB sky. So the analysis of [32] of the Laplace spectra and eigenfunctions uses the transition between these two bases. The resulting simulated CMB skies are suitable for an investigation for flat cosmic topologies with the "circles in the sky" method. A statistical analysis of distance correlations between cosmic sources, aimed at identifying possible signatures of cosmic topologies given by flat tori with the method of "cosmic crystallography" was performed in [19].

While an early analysis of the anomalies of the anisotropy spectrum of the CMB (the quadrupole suppression, the small value of the two-point temperature correlation function at large angles, and the quadrupole-octupole alignment) suggested that flat tori would account for all of these anomalies, if one of the sides of the fundamental domain is of the order of half the horizon scale, the more detailed analysis of [29] excludes this possibility on the basis of the "circles in the sky method" and of the $S$-statistic test, measuring reflection symmetry. Nonetheless, the flat tori remain at present one of the most promising possible candidates for multiconnected cosmic topologies.

An analysis of how to produce a quadrupole-octupole alignment for a flat torus with cubic fundamental domain, depending on the size $\ell$ of the 
torus, was given in [1. However, the alignment obtained in this way is not strong enough to account for the observed anomaly. Comparison with candidates such as dodecaredral and octahedral cosmologies shows that in these spherical topologies one has either no alignment or an anti-alignment, which appears to favor the flat tori.

We show here that, from the point of view of our model of gravity based on the spectral action functional, a cosmic topology given by a flat torus generates an inflation potential and slow-roll parameters that are different from those of the spherical topologies considered in the previous sections.

8.1. The spectral action on the flat tori. Let $T^{3}$ be the flat torus $\mathbb{R}^{3} / \mathbb{Z}^{3}$. The spectrum of the Dirac operator, denoted $D_{3}$, is given in Theorem 4.1 of [4] as

$$
\pm 2 \pi\left\|(m, n, p)+\left(m_{0}, n_{0}, p_{0}\right)\right\|
$$

where $(m, n, p)$ runs through $\mathbb{Z}^{3}$. Each value of $(m, n, p)$ contributes multiplicity 1 . The constant vector $\left(m_{0}, n_{0}, p_{0}\right)$ depends on the choice of spin structure.

Theorem 8.1. The spectral action $\operatorname{Tr}\left(f\left(D_{3}^{2} / \Lambda^{2}\right)\right)$ for the torus $T^{3}=\mathbb{R}^{3} / \mathbb{Z}^{3}$ is independent of the spin structure on $T^{3}$ and given by

$$
\operatorname{Tr}\left(f\left(D_{3}^{2} / \Lambda^{2}\right)\right)=\frac{\Lambda^{3}}{4 \pi^{3}} \int_{\mathbb{R}^{3}} f\left(u^{2}+v^{2}+w^{2}\right) d u d v d w+O\left(\Lambda^{-k}\right),
$$

for arbitrary $k>0$.

Proof. By (8.1), we know the spectrum of $D_{3}^{2}$ is given by

$$
4 \pi^{2}\left\|(m, n, p)+\left(m_{0}, n_{0}, p_{0}\right)\right\|^{2},
$$

where $(m, n, p)$ runs through $\mathbb{Z}^{3}$, and each value of $(m, n, p)$ contributes multiplicity 2.

Given a test function in Schwartz space, $f \in \mathcal{S}(\mathbb{R})$, the spectral action is then given by

$$
\operatorname{Tr}\left(f\left(D_{3}^{2} / \Lambda^{2}\right)\right)=\sum_{(m, n, p) \in \mathbb{Z}^{3}} 2 f\left(\frac{4 \pi^{2}\left(\left(m+m_{0}\right)^{2}+\left(n+n_{0}\right)^{2}+\left(p+p_{0}\right)^{2}\right)}{\Lambda^{2}}\right),
$$

In three dimensions, the Poisson summation formula is given by

$$
\sum_{\mathbb{Z}^{3}} g(m, n, p)=\sum_{\mathbb{Z}^{3}} \widehat{g}(m, n, p)
$$

where the Fourier transform is defined by

$$
\widehat{g}(m, n, p)=\int_{\mathbb{R}^{3}} g(u, v, w) e^{-2 \pi i(m u+n v+p w)} d u d v d w .
$$

If we define

$$
g(m, n, p)=f\left(\frac{4 \pi^{2}\left(\left(m+m_{0}\right)^{2}+\left(n+n_{0}\right)^{2}+\left(p+p_{0}\right)^{2}\right)}{\Lambda^{2}}\right),
$$


and apply the Poisson summation formula, we obtain the following expression for the spectral action:

$$
\begin{aligned}
\operatorname{Tr}\left(f\left(D_{3}^{2} / \Lambda^{2}\right)\right) & =2 \sum_{(m, n, p) \in \mathbb{Z}^{3}} \widehat{g}(m, n, p) \\
& =2 \widehat{g}(0,0,0)+O\left(\Lambda^{-k}\right) \\
& =2 \int_{\mathbb{R}^{3}} f\left(\frac{4 \pi^{2}\left(\left(u+m_{0}\right)^{2}+\left(v+n_{0}\right)^{2}+\left(w+p_{0}\right)^{2}\right)}{\Lambda^{2}}\right) d u d v d w \\
& +O\left(\Lambda^{-k}\right) \\
& =\frac{\Lambda^{3}}{4 \pi^{3}} \int_{\mathbb{R}^{3}} f\left(u^{2}+v^{2}+w^{2}\right) d u d v d w+O\left(\Lambda^{-k}\right)
\end{aligned}
$$

The estimate $\sum_{(m, n, p) \neq 0} \widehat{g}(m, n, p)=O\left(\Lambda^{-k}\right)$ for arbitrary $k>0$ is elementary, using the fact that $f \in \mathcal{S}(\mathbb{R})$. We observe that the nonperturbative spectral action is independent of the choice of spin structure.

Now let $X=T^{3} \times S_{\beta}^{1}$. We then compute the spectral action for the operator $D_{X}^{2}$ as a direct consequence of the previous result.

Theorem 8.2. On the 4-manifold $X=T^{3} \times S_{\beta}^{1}$, with the flat torus of size $\ell$ and with the product Dirac operator $D_{X}$ as in (4.3), the spectral action is given by

$$
\operatorname{Tr}\left(h\left(D_{X}^{2} / \Lambda^{2}\right)\right)=\frac{\Lambda^{4} \beta \ell^{3}}{4 \pi} \int_{0}^{\infty} u h(u) d u+O\left(\Lambda^{-k}\right)
$$

for arbitrary $k>0$.

Proof. For the operator $D_{X}^{2}$, with $D_{X}$ as in (4.3) the spectral action $\operatorname{Tr}\left(h\left(D_{X}^{2} / \Lambda^{2}\right)\right)$ is given by

$$
\sum_{(m, n, p, r) \in \mathbb{Z}^{4}} 2 h\left(\frac{4 \pi^{2}}{(\Lambda \ell)^{2}}\left(\left(m+m_{0}\right)^{2}+\left(n+n_{0}\right)^{2}+\left(p+p_{0}\right)^{2}\right)+\frac{1}{(\Lambda \beta)^{2}}\left(r+\frac{1}{2}\right)^{2}\right) .
$$

We set

$$
g(u, v, w, y)=2 h\left(\frac{4 \pi^{2}}{\Lambda^{2}}\left(u^{2}+v^{2}+w^{2}\right)+\frac{y^{2}}{(\Lambda \beta)^{2}}\right) .
$$

The Poisson summation formula then gives

$$
\sum_{(m, n, p, r) \in \mathbb{Z}^{4}} g\left(m+m_{0}, n+n_{0}, p+p_{0}, r+\frac{1}{2}\right)=\sum_{(m, n, p, r) \in \mathbb{Z}^{4}}(-1)^{r} \widehat{g}(m, n, p, r) .
$$

Since we have $h \in \mathcal{S}(\mathbb{R})$, we can estimate that the error term

$$
\sum_{(m, n, p, r) \neq(0,0,0,0)} \widehat{g}(m, n, p, r)
$$


is bounded by $O\left(\Lambda^{-k}\right)$ for arbitrary $k>0$. We then obtain

$$
\operatorname{Tr}\left(h\left(D_{X}^{2} / \Lambda^{2}\right)\right)=\widehat{g}(0,0,0,0)+O\left(\Lambda^{-k}\right) .
$$

We have

$$
\widehat{g}(0,0,0,0)=\int_{\mathbb{R}^{4}} 2 h\left(\frac{4 \pi^{2}}{(\Lambda \ell)^{2}}\left(u^{2}+v^{2}+w^{2}\right)+\frac{y^{2}}{(\Lambda \beta)^{2}}\right) d u d v d w d y .
$$

This gives

$\frac{\Lambda^{4} \beta \ell^{3}}{4 \pi^{3}} \int_{\mathbb{R}^{4}} h\left(u^{2}+v^{2}+w^{2}+y^{2}\right) d u d v d w d y=\frac{\Lambda^{4} \beta \ell^{3} \operatorname{Vol}\left(S^{3}\right)}{4 \pi^{3}} \int_{0}^{\infty} h\left(\rho^{2}\right) \rho^{3} d \rho$ which gives

$$
\operatorname{Tr}\left(h\left(D_{X}^{2} / \Lambda^{2}\right)\right)=\frac{\Lambda^{4} \beta \ell^{3}}{2 \pi} \int_{0}^{\infty} \rho^{3} h\left(\rho^{2}\right) d \rho+O\left(\Lambda^{-k}\right),
$$

from which we obtain (8.4).

We now consider the effect of introducing the perturbation $D^{2} \mapsto D^{2}+\phi^{2}$ in the spectral action. We write as above

$$
\mathcal{V}(x)=\int_{0}^{\infty} u(h(u+x)-h(u)) d u .
$$

We then have the following.

Theorem 8.3. The perturbed spectral action on the flat tori is of the form

$$
\operatorname{Tr}\left(h\left(\left(D_{X}^{2}+\phi^{2}\right) / \Lambda^{2}\right)\right)=\operatorname{Tr}\left(h\left(D_{X}^{2} / \Lambda^{2}\right)\right)+\frac{\Lambda^{4} \beta \ell^{3}}{4 \pi} \mathcal{V}\left(\phi^{2} / \Lambda^{2}\right) .
$$

The corresponding slow-roll potential is of the form

$$
V(\phi)=\frac{\Lambda^{4} \beta \ell^{3}}{4 \pi} \mathcal{V}\left(\phi^{2} / \Lambda^{2}\right),
$$

and the slow-roll parameters are given by

$$
\begin{gathered}
\epsilon=\frac{m_{P l}^{2}}{16 \pi}\left(\frac{\int_{x}^{\infty} h(u) d u}{\int_{0}^{\infty} u(h(u+x)-h(u)) d u}\right)^{2} \\
\eta=\frac{m_{P l}^{2}}{8 \pi}\left(\frac{h(x)}{\int_{0}^{\infty} u(h(u+x)-h(u)) d u}-\frac{1}{2}\left(\frac{\int_{x}^{\infty} h(u) d u}{\int_{0}^{\infty} u(h(u+x)-h(u)) d u}\right)^{2}\right) .
\end{gathered}
$$

Proof. The result follows directly from (8.4) upon writing

$$
\begin{gathered}
\operatorname{Tr}\left(h\left(\left(D_{X}^{2}+\phi^{2}\right) / \Lambda^{2}\right)\right)=\frac{\Lambda^{4} \beta \ell^{3}}{4 \pi} \int_{0}^{\infty} u h(u) d u+O\left(\Lambda^{-k}\right) \\
=\frac{\Lambda^{4} \beta \ell^{3}}{4 \pi} \int_{0}^{\infty} u(h(u+x)-h(u)) d u+\frac{\Lambda^{4} \beta \ell^{3}}{4 \pi} \int_{0}^{\infty} u h(u) d u+O\left(\Lambda^{-k}\right),
\end{gathered}
$$

and computing the slow-roll parameters as in (15.5) and (5.6). 
Notice how the absence of the $\mathcal{W}\left(\phi^{2} / \Lambda^{2}\right)$ term in the slow-roll potential for the case of flat tori gives rise to slow-roll parameters that are genuinely different from those we computed for the spherical geometries. This shows that, in noncommutative geometry models of gravity based on the spectral action functional, there is a nontrivial relation between cosmic topology (or at least the underlying curvature geometry) and the shape of the induced inflation slow-roll potential and parameters.

The case of the other flat geometries, the Bieberbach manifolds, can be handled with similar techniques, based on the explicit computation of their Dirac spectra given in [30].

\section{Geometric engineering of inflation scenarios via Dirac SPECTRA}

If one renounces the assumption of homogeneity and constant curvature, which reduces the candidate topologies to spherical and flat space forms, one finds that it is possible to engineer different inflation scenarios, by changing the slow-roll potential and the resulting slow roll parameters by modifying the metric on a fixed topology and change accordingly the Dirac spectrum and the resulting spectral action.

In the spherical examples we computed explicitly in the previous sections the Dirac spectra tend to have non-trivial multiplicities. These reflect the very symmetric form of the geometry. On the contrary, it is shown in [14] that, for a generic Riemannian metric on a given smooth compact 3 -dimensional manifold $M$, all the Dirac eigenvalues are simple.

Moreover, the result of [13] shows that, for a given $L>0$ and an assigned sequence of non-zero real numbers

$$
-L<\lambda_{1}<\lambda_{2}<\lambda_{3}<\cdots<\lambda_{N}<L,
$$

it is possible to construct, on an arbitrary smooth compact spin 3-manifold $M$, a Riemannian metric $g$ such that the non-zero spectrum of corresponding Dirac operator $D_{M}$ in the interval $(-L, L)$ consists of the simple eigenvalues

$$
\operatorname{Spec}\left(D_{M}\right) \cap((-L, L) \backslash\{0\})=\left\{\lambda_{j}\right\}_{j=1, \ldots, N} .
$$

The way to obtain a Dirac spectrum with these properties is to start with a metric on $M$ for which the dimension of the kernel of the Dirac operator is minimal, compatibly with the constraint given by the index theorem. By rescaling this metric one ensures that no other eigenvalue occurs in an interval $(-3 L, 3 L)$. One then performs a connected sum with $N$ copies of $S^{3}$, so that the resulting manifold is still topologically the same as $M$. One endows each of the 3 -spheres with a Berger metric as in [20], scaled so that the interval $(-2 L, 2 L)$ contains only one eigenvalue of the Dirac operator. Then applying a surgery formula one obtains the desired eigenvalues for the Dirac spectrum on the connected sum manifold, [13]. 
For simplicity, to avoid handling separately a possible kernel, let us consider here a variant of the spectral action where one only sums over the non-zero spectrum of $D$. We write this as

$$
\operatorname{Tr}^{\prime}(f(D / \Lambda)):=\sum_{\lambda \in \operatorname{Spec}(D) \backslash\{0\}} f(\lambda / \Lambda) .
$$

We then have the following result, which allows us to construct, on a given 3 -manifold a metric with prescribed spectral action.

Lemma 9.1. Let $M$ be a compact smooth 3-manifold, with a given spin structure. Let $f$ be a smooth function, compactly supported inside an interval $[-L / \Lambda, L / \Lambda]$. Then, for any given $\lambda>0$, there is a metric $g_{\lambda, L}$ on $M$ such that the spectral action for the resulting Dirac operator is

$$
\operatorname{Tr}^{\prime}(f(D / \Lambda))=\frac{\Lambda}{\lambda} \hat{f}(0)+O\left(\Lambda^{-k}\right)
$$

for arbitrary $k>0$.

Proof. Let $\lambda_{n}=\eta+n \lambda$ be a progression indexed by the integers $n \in \mathbb{Z}$, with $\lambda>0$ and $\eta \neq 0$. Let $\left\{\lambda_{n_{0}+j}\right\}_{j=1, \ldots, N}$ be the points of this sequence that lie in the interval $(-L, L)$. We assume that $\lambda_{n} \neq \pm L$ for all $n$. Using the method of [13] we construct, by taking connected sums with Berger spheres, a metric on $M$ for which the Dirac operator $D$ has $\operatorname{Spec}(D) \cap((-L, L) \backslash\{0\})$ given by the simple eigenvalues $\lambda_{n_{0}+j}$, with $j=1, \ldots, N$. For a test function supported in $[-L / \Lambda, L / \Lambda]$ we then have

$$
\operatorname{Tr}^{\prime}(f(D / \Lambda))=\sum_{n \in \mathbb{Z}} f\left(\lambda_{n} / \Lambda\right)
$$

We can then use the Poisson summation formula

$$
\sum_{n \in \mathbb{Z}} g(\eta+n \lambda)=\sum_{n \in \mathbb{Z}} \frac{1}{\lambda} \exp \left(\frac{2 \pi i n \eta}{\lambda}\right) \widehat{g}\left(\frac{n}{\lambda}\right)
$$

to $g(u)=f(u / \Lambda)$. We estimate as in the previous cases that

$$
\left|\sum_{n \neq 0} \frac{1}{\lambda} \exp \left(\frac{2 \pi i n \eta}{\lambda}\right) \widehat{g}\left(\frac{n}{\lambda}\right)\right| \leq O\left(\Lambda^{-k}\right),
$$

for arbitrary $k>0$, so that we are left with the term

$$
\frac{1}{\lambda} \widehat{g}(0)=\frac{\Lambda}{\lambda} \widehat{f}(0)
$$

Notice, for example, that we can apply this result starting from any one of the spherical topologies we analyzed in the previous sections. In such cases one starts with the round metric, so one does not have a kernel to worry about. One then scales it so as not to have any other eigenvalue in the interval $(-3 L, 3 L)$ and proceeds to modify the metric by taking connected sums with the Berger spheres to insert the desired eigenvalues in the interval 
$(-L, L)$. Thus, on a given underlying topology one can significantly alter the form of the spectral action by this method, at the cost of no longer having a homogeneous metric. We now show the effect this operation has on the inflation slow-roll potential, even though such non-homogeneous metrics are clearly less interesting in terms of candidate cosmologies.

We now see how this procedure can be used to construct different possible slow-roll potentials.

Let $\left(\lambda_{n}, P\right)$ denote the following data:

- A progression $\lambda_{n}=\eta+n \lambda$, for $n \in \mathbb{Z}$, with $\lambda>0$ and $\eta \neq 0$.

- A polynomial $P(u)=\alpha u^{2}+\gamma$ with the property that $P\left(\lambda_{n}\right)=m_{n}$ is a non-negative integer, for all $n$.

In the following, as above, we assume that either we start from a manifold $M$ with a metric for which $D_{M}$ has trivial kernel, or else we modify the spectral action on $M \times S^{1}$ to count only the non-zero part of the spectrum of $D_{M}$.

Proposition 9.2. Let $M$ be a compact smooth 3-dimensional manifold endowed with a spin structure. Given a smooth compactly supported test function $h$ such that $h \equiv 1$ on an interval $[-T, T]$ and decays rapidly to zero outside of this interval, and given a choice of data $\left(\lambda_{n}, P\right)$ as above, there exists a Riemannian metric $g$ on $M$ such that the resulting Dirac operator $D$ of the form (4.3) on $M \times S_{\beta}^{1}$ has spectral action

$$
\operatorname{Tr}\left(h\left(D^{2} / \Lambda^{2}\right)\right)=\frac{\pi \Lambda^{4} \beta \alpha}{\lambda} \int_{0}^{\infty} u h(u) d u+\frac{2 \pi \Lambda^{2} \beta \gamma}{\lambda} \int_{0}^{\infty} h(u) d u+O\left(\Lambda^{-k}\right),
$$

for arbitrary $k>0$.

Proof. Consider the sequence of non-negative integers $m_{n}=P\left(\lambda_{n}\right)$. Let $\Omega \subset \mathbb{Z}^{2}$ be the set of pairs $(n, m)$ such that

$$
x_{n, m}(\Lambda, \beta):=\frac{\lambda_{n}^{2}}{\Lambda^{2}}+\frac{(m+1 / 2)^{2}}{(\Lambda \beta)^{2}} \in(0, T) .
$$

We can assume that all other points of the form $x_{n, m}$, for $(n, m) \notin \Omega$ lie outside of the support of $h$. Let $(-L, L)$ be an interval that contains all the points $\lambda_{n}$ for which the set of $m \in \mathbb{Z}$ with $(n, m) \in \Omega$ is non-empty. For all $n \in \mathbb{Z}$ such that the set of $(n, m) \in \Omega$ is nonempty, choose sufficiently small, non-intersecting open intervals $U_{n, \epsilon}=\left(\lambda_{n}-\epsilon, \lambda_{n}+\epsilon\right)$ around the value $\lambda_{n}$, such that all $\lambda \in U_{n, \epsilon}$ have the property that $\frac{\lambda^{2}}{\Lambda^{2}}+\frac{(m+1 / 2)^{2}}{(\Lambda \beta)^{2}} \in(0, T)$, for all $m$ such that $(n, m) \in \Omega$. Then choose $m_{n}$ points $\lambda_{n, 1}<\lambda_{n, 2}<$ $\cdots<\lambda_{n, m_{n}}$ inside $U_{n, \epsilon}$. By the construction of [13], by taking connected sums with suitable Berger spheres, we can obtain on $M$ a metric for which the Dirac operator $D_{M}$ has spectrum satisfying $\operatorname{Spec}\left(D_{M}\right) \cap((-L, L) \backslash$ $\{0\})=\left\{\lambda_{n, j}\right\}$ with $n=n_{0}, \ldots, n_{0}+N$ and $j=1, \ldots, m_{n}$. Let $D$ be the corresponding Dirac operator (4.3) on $M \times S_{\beta}^{1}$. The spectral action 
$\operatorname{Tr}\left(h\left(D^{2} / \Lambda^{2}\right)\right)$ is computed by

$$
\sum_{n, j} 2 h\left(\lambda_{n, j}^{2} \Lambda^{-2}+(m+1 / 2)^{2}(\Lambda \beta)^{-2}\right)
$$

Given the construction of the $\lambda_{n, j}$ above, this is also equal to

$$
\sum_{(n, m) \in \mathbb{Z}^{2}} 2 P\left(\lambda_{n}\right) h\left(\lambda_{n}^{2} \Lambda^{-2}+(m+1 / 2)^{2}(\Lambda \beta)^{-2}\right) .
$$

We let $g(u, v)=2 P(u) h\left(u^{2} \Lambda^{-2}+v^{2}(\Lambda \beta)^{-2}\right)$ and we obtain, by the Poisson summation formula,

$$
\sum_{(n, m) \in \mathbb{Z}^{2}} g\left(n \lambda+\eta, m+\frac{1}{2}\right)=\sum_{(n, m) \in \mathbb{Z}^{2}}(-1)^{m} \exp \left(\frac{2 \pi i n \eta}{\lambda}\right) \frac{1}{\lambda} \widehat{g}\left(\frac{n}{\lambda}, m\right) .
$$

Estimating as before the sum of terms with $(n, m) \neq(0,0)$ to be bounded by $O\left(\Lambda^{-k}\right)$ for arbitrary $k>0$, this gives

$$
\operatorname{Tr}\left(h\left(D^{2} / \Lambda^{2}\right)\right)=\frac{1}{\lambda} \widehat{g}(0,0)+O\left(\Lambda^{-k}\right),
$$

where we then have

$$
\begin{gathered}
\widehat{g}(0,0)=\int_{\mathbb{R}^{2}} 2\left(\alpha u^{2}+\gamma\right) h\left(u^{2} \Lambda^{-2}+v^{2}(\Lambda \beta)^{-2}\right) d u d v \\
=\Lambda^{4} \beta \alpha \int_{\mathbb{R}^{2}}\left(u^{2}+v^{2}\right) h\left(u^{2}+v^{2}\right) d u d v+2 \Lambda^{2} \beta \gamma \int_{\mathbb{R}^{2}} h\left(u^{2}+v^{2}\right) d u d v \\
=\Lambda^{4} \beta \alpha 2 \pi \int_{0}^{\infty} \rho^{3} h\left(\rho^{2}\right) d \rho+\Lambda^{2} \beta \gamma 4 \pi \int_{0}^{\infty} h\left(\rho^{2}\right) d \rho \\
=\Lambda^{4} \beta \alpha \pi \int_{0}^{\infty} u h(u) d u+\Lambda^{2} \beta \gamma 2 \pi \int_{0}^{\infty} h(u) d u .
\end{gathered}
$$

We then have the following result, which shows that altering the spatial metric on suitable bubbles (the Berger spheres with which one performs a connected sum) consequently alters the form of the inflation potential and slow-roll parameters induced by the spectral action.

Corollary 9.3. For a 3-manifold $M$ with a metric constructed as in Proposition 9.2 above, the induced slow-roll potential has the form

$$
V(\phi)=\frac{\pi \Lambda^{4} \alpha \beta}{\lambda} \mathcal{V}\left(\phi^{2} / \Lambda^{2}\right)-\frac{2 \pi \Lambda^{2} \gamma \beta}{\lambda} \mathcal{W}\left(\phi^{2} / \Lambda^{2}\right),
$$

with $\mathcal{V}$ and $\mathcal{W}$ as in (5.3). The corresponding slow-roll parameters are given by

$$
\begin{gathered}
\epsilon(x)=\frac{m_{P l}^{2}}{16 \pi}\left(\frac{\alpha \Lambda^{2} \mathcal{V}^{\prime}(x)-2 \gamma \mathcal{W}^{\prime}(x)}{\alpha \Lambda^{2} \mathcal{V}(x)-2 \gamma \mathcal{W}(x)}\right)^{2} \\
\eta(x)=\frac{m_{P l}^{2}}{8 \pi}\left(\frac{\alpha \Lambda^{2} \mathcal{V}^{\prime \prime}(x)-2 \gamma \mathcal{W}^{\prime \prime}(x)}{\alpha \Lambda^{2} \mathcal{V}(x)-2 \gamma \mathcal{W}(x)}-\frac{1}{2}\left(\frac{\alpha \Lambda^{2} \mathcal{V}^{\prime}(x)-2 \gamma \mathcal{W}^{\prime}(x)}{\alpha \Lambda^{2} \mathcal{V}(x)-2 \gamma \mathcal{W}(x)}\right)^{2}\right) .
\end{gathered}
$$


Proof. This follows directly from the previous result, by computing $\operatorname{Tr}\left(h\left(D^{2}+\right.\right.$ $\left.\phi^{2}\right) / \Lambda^{2}$ ) in the same way as in the previous cases.

Notice that, after rotating back to Lorentzian signature with a metric of the Friedmann form (5.4), the factor $\Lambda$ in the slow-roll parameter appears in fact multiplied by the scale factor $a(t)$ of the Friedmann metric, which gives a constant term by the relation $\Lambda(t) \sim 1 / a(t)$, as in the previous cases. One is left with the freedom of modifying the slow-roll parameters by changing the modified metric on the Berger spheres and correspondingly affecting the values of the parameters $\alpha$ and $\gamma$.

One can also obtain potentials of a more general form, if one constructs, via the same method, spectra that are only partially given by arithmetic progressions. An example of this sort is computed explicitly in the appendix: it gives rise to a genuinely different shape of the potential $V(\phi)$.

\section{Conclusions}

In models of high-energy physics based on noncommutative geometry, the spectral action functional of [8] is proposed as an action functional for gravity, or for gravity coupled to matter when additional noncomumutative extra dimensions are introduced in the geometry of the model. We concentate here on the purely gravitational part of the model, without noncomumutative extra dimensions, and we compute the explicit nonperturbative form of the spectral action functional for three among the more likely candidates for the problem of cosmic topology: the quaternionic space $S U(2) / Q 8$ and the Poincaré dodecahedral space $S^{3} / \Gamma$, with $\Gamma$ the binary icosahedral group, and the flat tori. We show that when one computes the spectral action for the 4-dimensional manifold obtained by Wick rotating and compactifying the corresponding space-time to a product of the given 3-manifold by a circle, one obtains as non-perturbative effect a slow-roll potential for a field $\phi$ coming from perturbations $D^{2} \mapsto D^{2}+\phi$ of the Dirac operator of the 4-dimensional geometry. We compute the slow-roll parameters for the resulting slow-roll potential $V(\phi)$ and show that they make sense when rotating back to the original Minkowskian spacetime. We see that, in the case of the quaternionic and the dodecahedral space, the slow-roll parameters are the same as for the ordinary case of the sphere $S^{3}$, while in the case of the flat tori the potential one obtains in this way behaves significantly differently from the spherical cases. This shows that cosmological models based on noncommutative geometry predict that different candidate cosmic topologies may give rise to different inflation scenarios, and different values for testable cosmological parameters. 


\section{Appendix: Lens spaces, a false positive}

Lens spaces are quotients of the sphere $S^{3}$ by the action of a finite cyclic group $\mathbb{Z} / N \mathbb{Z}$. They have been considered among the candidate cosmic topologies, especially in [38], which shows simulated CMB maps for lens spaces and computes the expected CMB anisotropies for some of these topologies. The surprising result of the analysis of [38 is that instead of finding an increasingly suppressed quadrupole with increasing $N$, the low multipoles are enhanced instead of being suppressed for large $N$ Thus, the simulated power spectra of 38] suggest that to maintain consistency with the WMAP data, one cannot exceed the range $N \leq 15$. On the other hand, in the same work 38] the lens space case is analyzed from the point of view of the "circles in the sky" method and it is shown that potentially detectable periodicities (matching circles) would appear only in the range $N>7$.

11.1. The trouble with the Dirac spectrum on lens spaces. Consider in particular lens spaces $\mathcal{L}_{N}=S U(2) / \mathbb{Z}_{N}$, with $N \geq 3$, which are quotients of the sphere $S U(2)=S^{3}$ by the action of the finite cyclic group $\mathbb{Z}_{N}=\mathbb{Z} / N \mathbb{Z}$ acting on $S U(2) \subset \mathbb{C}^{2}$ by

$$
\left(\begin{array}{cc}
\omega & 0 \\
0 & \omega^{-1}
\end{array}\right), \quad \text { with } \quad \omega^{N}=1 .
$$

For these lens spaces, Bär gave an explicit computation of the Dirac spectrum in [3]. The result states that, for the canonical spin structure, the spectrum is of the form

(i) $\quad-i N-\frac{1}{2}$, with multiplicity $2 i N, i=0,1,2, \ldots$

(ii) $-\frac{1}{2} \pm m$, with multiplicity $m$,

$$
m=2,3, \ldots,-(m+1)<i N \leq(m-2)
$$

where we have taken on the sphere $S^{3}$ the round metric of radius one. In the even case $N=2 N^{\prime}$, there is also a second spin structure for which the Dirac spectrum is given in [3] as

(i) $\quad-\left(N^{\prime}+i N\right)-\frac{1}{2}$ with multiplicity $2\left(N^{\prime}+i N\right), i=0,1,2, \ldots$

(ii) $-\frac{1}{2} \pm m$ with multiplicity $m$,

$m=2,3, \ldots \quad 1-m<i N+\frac{N}{2} \leq m-2$.

Unfortunately, this result of [3] appears to be incorrect, as we discuss in $\$ 11.7$ below.

However, we still show here what the spectral action and slow-roll potential would be for a manifold with Dirac spectrum as above, because the computation itself exhibits some interesting features that we have not encountered in the other spherical and flat examples and that may be useful in different contexts, for manifolds whose Dirac spectrum only partially decomposes as a union of arithmetic progressions. 
We show that the incorrect calculation of the Dirac spectrum of $\mathcal{L}_{N}$ of [3] leads to a "false positive" result of a spherical cosmic topology which gives rise to an inflation scenario different from the simply connected case. However, as we show in 11.7 below, with the correct calculation of the Dirac spectrum for the lens spaces, the inflation potential is in fact again the same as for the case of the sphere, just as in the other spherical cases we computed in this paper.

11.2. Multiplicities, first case. We consider here the problem of computing the explicit nonperturbative form of the spectral action for an operator $D$ with spectrum of the form (11.2).

We start by writing the multiplicities in a more convenient form. The multiplicity in row $(i)$ is already in a nice form. To handle row $(i i)$, we need to break it up into the subsets corresponding to each equivalence class $m \equiv j(\bmod N)$, where $j \in\{0,1, \ldots, N-1\}$.

In order to determine the multiplicity of $-1 / 2 \pm m$, it is convenient to replace the upper and lower bounds of $-(m+1)<i N \leq(m-2)$ with the smallest and largest values of $i N$ which satisfy the inequality.

Lemma 11.1. The multiplicity of $-1 / 2 \pm m$ is given by

$$
\begin{array}{lll}
\frac{2 m(m-j)}{N} & \text { for } m \equiv j \quad \bmod N, \text { with } j=0,1 \\
\frac{2 m^{2}-2 m j+m N}{N} & \text { for } m \equiv j & \bmod N, \text { with } j=2,3, \ldots, N-1 .
\end{array}
$$

Proof. We first look at the case where $m \equiv j(\bmod N), j=0,1$. In this case, the bound $-m-1<i N \leq m-2$ can be replaced by

$$
j-m \leq i N \leq m-j-N,
$$

by adding $j+1$ to the left hand side and subtracting $N+j-2$ from the right hand side. What matters is that $0<j+1 \leq N$, and $0 \leq N+j-2<N$.

If $m=k N+j$, where $k=0,1,2, \ldots$, then we see that there are $2 k$ values of $i$ which satisfy the inequality, and hence $-1 / 2 \pm m$ has multiplicity $2 k m=(2 m(m-j)) / N$. Notice that when $m$ is 0 or 1 , this formula gives us a multiplicity of zero, which is good, since in row $(i i)$, the index $m$ begins at $m=2$.

We then consider the case $m \equiv j(\bmod N), j=2,3, \ldots, N-1$. One can replace the upper and lower bounds $-m-1<i N \leq m-2$ by

$$
j-m \leq i N \leq m-j,
$$

by adding $j+1$ to the left hand side, and subtracting $j-2$ from the right hand side. One has $0<j+1 \leq N$, and $0 \leq j-2<N$.

If $m=k N+j, k=0,1,2, \ldots$, then we see that there are $2 k+1$ values of $i$ which satisfy the inequality, and hence $-1 / 2 \pm m$ has multiplicity $(2 k+1) m=$ $\left(2 m^{2}-2 m j+m N\right) / N$. 
11.3. The spectral action and the Poisson formula. One sees then that, unlike the cases of the spherical topologies we analyzed before, one cannot simply write the whole spectrum (11.2) as a union of arithmetic progressions indexed over the integers. However, it is still possible to extend the positive and the negative part of the spectrum, separately, to unions of such arithmetic progressions.

This provides us with a different method, still based on the Poisson summation formula, to compute the spectral action, which may turn out to be useful in other cases. This is our main reason for including the full calculation here, despite the fact that it does not give the correct answer for lens spaces.

One finds that the multiplicities, for the positive and the negative parts of the spectrum, can be interpolated by polynomials, in the following way.

Lemma 11.2. For $m>0$, the multiplicity of $-1 / 2+m$, when $m \equiv i \bmod$ $N$, is given by $P_{i}^{+}(-1 / 2+m)$, with $P_{i}^{+}$the polynomials

$$
\begin{aligned}
& P_{0}^{+}(u)=\frac{2}{N} u^{2}+\frac{2}{N} u+\frac{1}{2 N} \\
& P_{1}^{+}(u)=\frac{2}{N} u^{2}-\frac{1}{2 N} \\
& P_{j}^{+}(u)=\frac{2}{N} u^{2}+\frac{2-2 j+N}{N} u+\frac{1-2 j+N}{2 N}, \quad j=2,3, \ldots, N-1,
\end{aligned}
$$

For $m \geq 0$, the multiplicity of $-1 / 2-m$, when $m \equiv \ell \bmod N$ is given by $P_{\ell}^{-}(-1 / 2-m)$, with $P_{\ell}^{-}$the polynomial

$$
\begin{aligned}
& P_{0}^{-}(u)=\frac{2}{N} u^{2}+\frac{2}{N} u+\frac{1}{2 N} \\
& P_{1}^{-}(u)=\frac{2}{N} u^{2}+\frac{4}{N} u+\frac{3}{2 N} \\
& P_{j}^{-}(u)=\frac{2}{N} u^{2}+\frac{2+2 j-N}{N} u+\frac{1+2 j-N}{2 N}, \quad j=2,3, \ldots, N-1 .
\end{aligned}
$$

The multiplicity of $-i N-1 / 2$, for $i \geq 0$, is given by $P^{-}(-i N-1 / 2)$, with

$$
P^{-}(u)=-2 u-1 \text {. }
$$

Proof. This follows directly from the expressions for the multiplicities given in Lemma 11.1 above.

One can then make the following observation on computing the spectral action.

Lemma 11.3. Let $D$ be an operator with spectrum (11.2). Given a Schwartz function $f$, there are Schwartz functions $f_{+}$and $f_{-}$, respectively supported on the positive and negative reals, with the property that $f=f_{+}+f_{-}$on $(-\infty,-\alpha] \cup[\alpha, \infty)$, with $I_{\alpha}=(-\alpha, \alpha)$ an interval with $\operatorname{Spec}(D) \cap I_{\alpha}=\emptyset$. The spectral action for $D$ is then computed by

$$
\operatorname{Tr}(f(D / \Lambda))=\operatorname{Tr}\left(f_{+}(D / \Lambda)\right)+\operatorname{Tr}\left(f_{-}(D / \Lambda)\right) .
$$

Proof. One observes from (11.2) that there is a gap in the spectrum of $D$ around zero. Thus, it is possible to replace the function $f$ with a pair of Schwartz functions $f_{+}$and $f_{-}$, which are, respectively, equal to $f$ on the 
positive and negative parts of the spectrum and that have support contained only in the positive or negative reals. Since the values of $f$ and $f_{+}+f_{-}$on an open neighborhood of the spectrum are the same, the value of the spectral action is unchanged.

We can now compute the two terms on the right hand side of (11.8).

Theorem 11.4. Let $f_{+}$be a Schwartz function supported on the positive reals, chosen as in Lemma 11.3. Then, for an operator $D$ with spectrum of the form (11.2) one has

$$
\operatorname{Tr}\left(f_{+}(D / \Lambda)\right)=\frac{1}{N}\left(2 \Lambda^{3} \widehat{f}_{+}^{(2)}(0)+\Lambda^{2} \widehat{f}_{+}^{(1)}(0)\right)+\epsilon_{+}(\Lambda)
$$

where the error term is of order $\epsilon_{+}(\Lambda)=O\left(\Lambda^{-k}\right)$, for any $k>0$, and where $\hat{f}_{+}^{(k)}$ is the Fourier transform of $v^{k} f_{+}(v)$, as above.

Proof. We define $g_{j}^{+}(u)=P_{j}^{+}(u) f_{+}(u / \Lambda)$, with $P_{j}^{+}$as in (11.5), for $j=$ $0, \ldots, N-1$, so that we can write the spectral action with test function $f_{+}$ in the form

$$
\operatorname{Tr}\left(f_{+}(D / \Lambda)\right)=\sum_{k \in \mathbb{Z}} \sum_{j=0}^{N-1} g_{j}^{+}(-1 / 2+k N+j) .
$$

In fact, extending the sum to $m \in \mathbb{Z}$ does not change anything, since all terms with $m \leq 0$ fall outside of the support of $f_{+}$. We can then apply the Poisson summation formula to compute this expression. The analog of (6.6) now gives

$$
\sum_{k \in \mathbb{Z}} g_{j}^{+}(k N+(2 j-1) / 2)=\sum_{k \in \mathbb{Z}} \frac{1}{N} \exp \left(\frac{(2 j-1) \pi i k}{N}\right) \widehat{g}_{j}^{+}\left(\frac{k}{N}\right)
$$

The same argument used in [9] to estimate the remainder term applies here to give, for any $k>0$,

$$
\sum_{k \neq 0} \frac{1}{N}\left|\widehat{g}_{j}^{+}\left(\frac{k}{N}\right)\right| \leq O\left(\Lambda^{-k}\right)
$$

so that (11.10) can then be written as

$$
\operatorname{Tr}\left(f_{+}(D / \Lambda)\right)=\frac{1}{N} \sum_{j=0}^{N-1} \widehat{g}_{j}^{+}(0)+O\left(\Lambda^{-k}\right)
$$


We then obtain the following values for $\widehat{g}_{j}^{+}(0)$, using the form (11.5) of the polynomials $P_{j}^{+}$:

$$
\begin{aligned}
\widehat{g}_{0}^{+}(0)= & \frac{2}{N} \Lambda^{3} \widehat{f}_{+}^{(2)}(0)+\frac{2}{N} \Lambda^{2} \widehat{f}_{+}^{(1)}(0)+\frac{1}{2 N} \Lambda \widehat{f}_{+}(0) \\
\widehat{g}_{1}^{+}(0)= & \frac{2}{N} \Lambda^{3} \widehat{f}_{+}^{(2)}(0)-\frac{1}{2 N} \Lambda \widehat{f}_{+}(0) \\
\widehat{g}_{j}^{+}(0)= & \frac{2}{N} \Lambda^{3} \widehat{f}_{+}^{(2)}(0)+\frac{2-2 j+N}{N} \Lambda^{2} \widehat{f}_{+}^{(1)}(0)+\frac{1-2 j+N}{2 N} \Lambda \widehat{f}_{+}(0), \\
& j=2,3, \ldots, N-1
\end{aligned}
$$

This then gives

$$
\frac{1}{N} \sum_{j=0}^{N-1} \widehat{g_{j}^{+}}(0)=\frac{1}{N}\left(2 \Lambda^{3} \widehat{f}_{+}^{(2)}(0)+\Lambda^{2} \widehat{f}_{+}^{(1)}(0)\right),
$$

while the terms with $\widehat{f}_{+}(0)$ in this case add up to zero, since

$$
\frac{2}{N}+\sum_{j=2}^{N-1} \frac{2-2 j+N}{N}=1, \quad \text { and } \quad \sum_{j=2}^{N-1} \frac{1-2 j+N}{2 N}=0 .
$$

The argument for the term with $f_{-}$is similar. We have the following result.

Theorem 11.5. Let $f_{-}$be a Schwartz function supported on the negative reals, chosen as in Lemma 11.3. Then, for $D$ an operator with spectrum (11.2) one has

$$
\operatorname{Tr}\left(f_{-}(D / \Lambda)\right)=\frac{1}{N}\left(2 \Lambda^{3} \widehat{f}_{-}^{(2)}(0)+\Lambda^{2} \widehat{f}_{-}^{(1)}(0)\right)+\epsilon_{-}(\Lambda)
$$

with the error term $\epsilon_{-}(\Lambda)=O\left(\Lambda^{-k}\right)$, for any $k>0$.

Proof. We set $g_{j}^{-}(u)=P_{j}^{-}(u) f_{-}(u / \Lambda)$, with $P_{j}^{-}$as in (11.6), and $g^{-}(u)=$ $P^{-}(u) f_{-}(u / \Lambda)$, with $P^{-}$as in (11.7). Then we can write the spectral action on $\mathcal{L}_{N}$, with the Schwartz function $f_{-}$, in the form

$$
\operatorname{Tr}\left(f_{-}(D / \Lambda)\right)=\sum_{k \in \mathbb{Z}}\left(g^{-}(k N-1 / 2)+\sum_{j=0}^{N-1} g_{j}^{-}(-1 / 2+k N-j)\right)
$$

Then one can again use the Poisson summation formula

$$
\sum_{k \in \mathbb{Z}} g_{j}^{-}(k N-(2 j+1) / 2)=\sum_{k \in \mathbb{Z}} \frac{1}{N} \exp \left(\frac{-(2 j+1) \pi i k}{N}\right) \widehat{g}_{j}^{-}\left(\frac{k}{N}\right)
$$

and

$$
\sum_{k \in \mathbb{Z}} g^{-}(k N-1 / 2)=\sum_{k \in \mathbb{Z}} \frac{1}{N} \exp \left(\frac{-\pi i k}{N}\right) \widehat{g}^{-}\left(\frac{k}{N}\right)
$$


and an estimate of the error terms

$$
\sum_{k \neq 0} \frac{1}{N}\left|\widehat{g}_{j}^{-}\left(\frac{k}{N}\right)\right| \leq O\left(\Lambda^{-k}\right) \quad \text { and } \quad \sum_{k \neq 0} \frac{1}{N}\left|\widehat{g}^{-}\left(\frac{k}{N}\right)\right| \leq O\left(\Lambda^{-k}\right),
$$

as in [9] to write (11.15) as

$$
\operatorname{Tr}\left(f_{-}(D / \Lambda)\right)=\frac{1}{N}\left(\widehat{g}^{-}(0)+\sum_{j=0}^{N-1} \widehat{g}_{j}^{-}(0)\right)+O\left(\Lambda^{-k}\right) .
$$

One can then compute these values using the explicit form of the polynomials $P_{j}^{-}$and $P^{-}$of (11.6) and (11.7) and one obtains

$$
\begin{aligned}
\widehat{g}_{0}^{-}(0)= & \frac{2}{N} \Lambda^{3} \widehat{f}_{-}^{(2)}(0)+\frac{2}{N} \Lambda^{2} \widehat{f}_{-}^{(1)}(0)+\frac{1}{2 N} \Lambda \widehat{f}_{-}(0) \\
\widehat{g}_{1}^{-}(0)= & \frac{2}{N} \Lambda^{3} \widehat{f}_{-}^{(2)}(0)+\frac{4}{N} \Lambda^{2} \widehat{f}_{-}^{(1)}(0)+\frac{3}{2 N} \Lambda \widehat{f}_{-}(0) \\
\widehat{g}_{j}^{-}(0)= & \frac{2}{N} \Lambda^{3} \widehat{f}_{-}^{(2)}(0)+\frac{2+2 j-N}{N} \Lambda^{2} \widehat{f}_{-}^{(1)}(0)+\frac{1+2 j-N}{2 N} \Lambda \widehat{f}_{-}(0), \\
& j=2,3, \ldots, N-1 \\
\widehat{g}^{-}(0)= & -2 \Lambda^{2} \widehat{f}_{-}^{(1)}(0)-\Lambda \widehat{f}_{-}(0) .
\end{aligned}
$$

Thus, since

$\frac{2}{N}+\frac{4}{N}+\sum_{j=2}^{N-1} \frac{2+2 j-N}{N}-2=1$ and $\frac{1}{2 N}+\frac{3}{2 N}+\sum_{j=2}^{N-1} \frac{1+2 j-N}{2 N}-1=0$,

one then has

$$
\widehat{g}^{-}(0)+\sum_{j=0}^{N-1} \widehat{g}_{j}^{-}(0)=2 \Lambda^{3} \widehat{f}_{-}^{(2)}(0)+\Lambda^{2} \widehat{f}_{-}^{(1)}(0) .
$$

Thus, one obtains (11.14).

This gives a complete nonperturbative calculation of the spectral action as follows.

Theorem 11.6. The spectral action for an operator $D$ with spectrum (11.2) is given by

$$
\operatorname{Tr}(f(D / \Lambda)) \sim \frac{1}{N}\left(2 \Lambda^{3}\left(\widehat{f}_{+}^{(2)}(0)+\widehat{f}_{-}^{(2)}(0)\right)+\Lambda^{2}\left(\widehat{f}_{+}^{(1)}(0)+\widehat{f}_{-}^{(1)}(0)\right)\right),
$$

up to an error term of the order of $O\left(\Lambda^{-k}\right)$.

Proof. This follows directly from Lemma 11.3 and Theorems 11.4 and 11.5 . 
In particular, one is especially interested in the case where the function $f$ is a Schwartz function that approximates a cutoff function on an interval $[-\alpha, \alpha]$. In this case, $f$ is an even function and one can assume that the two functions $f_{+}$and $f_{-}$can be chosen to be mirror images, so that $f_{+}(x)=$ $f_{-}(-x)$.

Corollary 11.7. Let $f$ be an even Schwartz function such that the $f_{+}$and $f_{-}$of Lemma 11.3 satisfy $f_{+}(x)=f_{-}(-x)$. Then the spectral action of Theorem 11.6 is given by

$$
\operatorname{Tr}(f(D / \Lambda))=\frac{4}{N} \Lambda^{3} \widehat{f}_{+}^{(2)}(0)+O\left(\Lambda^{-k}\right) .
$$

Proof. The function $\widehat{f}_{ \pm}^{(k)}$ is the Fourier transform of $v^{k} f_{ \pm}(v)$, so that

$$
\widehat{f}_{ \pm}^{(k)}(0)=\int_{\mathbb{R}} v^{k} f_{ \pm}(v) d v=\int_{\mathbb{R}_{ \pm}} v^{k} f_{ \pm}(v) d v .
$$

Using $f_{+}(v)=f_{-}(-v)$ one sees that

$$
\int_{0}^{\infty} v^{2} f_{+}(v) d v=\int_{-\infty}^{0} v^{2} f_{-}(v) d v
$$

so that $\widehat{f}_{+}^{(2)}(0)=\widehat{f}_{-}^{(2)}(0)$, while

$$
\int_{0}^{\infty} v f_{+}(v) d v=-\int_{-\infty}^{0} v f_{-}(v) d v
$$

so that $\hat{f}_{+}^{(1)}(0)=-\widehat{f}_{-}^{(1)}(0)$. The $\Lambda^{2}$-terms then cancel.

11.4. The other spectrum. We also show, in a similar way, how one can compute the spectral action for an operator $D$ whose spectrum is of the form given in (11.3). As before, the multiplicity in row $(i)$ is already in a nice form, while for row $(i i)$ we obtain the following.

Lemma 11.8. The multiplicity of $-\frac{1}{2} \pm m$ in (11.3) is given by

$$
\begin{array}{lll}
\frac{2 m(m-j)}{N} & \text { for } m \equiv j \bmod N, \quad j=0,1, \ldots, \frac{N}{2}+1 \\
\frac{2 m(m-j+N)}{N} & \text { for } m \equiv j \quad \bmod N, \quad j=\frac{N}{2}+2, \ldots, N-1 .
\end{array}
$$

Proof. To handle row $(i i)$, we need to break it up into the pieces $m \equiv j$ $(\bmod N)$, where $j \in\{0,1, \ldots, N-1\}$. Similar to the previous spectrum, to find nice expressions for the multiplicities, it will be convenient to replace the upper and lower bounds with the highest and lowest values of $i N+\frac{N}{2}$ that satisfy the inequality.

We first consider the case with $m \equiv j(\bmod N), j=0,1, \ldots, N / 2+1$. The bound $1-m<i N+\frac{N}{2} \leq m-2$ can be replaced by

$$
\frac{N}{2}+j-m \leq i N+\frac{N}{2} \leq m-j-\frac{N}{2}
$$


by adding $\frac{N}{2}+j-1$ to the left hand side and subtracting $j-2+\frac{N}{2}$ from the right hand side. We check that $0<\frac{N}{2}+j-1 \leq N$, and that $0 \leq$ $j-2+\frac{N}{2}<N$. If $m=k N+j$, where $k=0,1,2, \ldots$, then we see that there are $2 k$ values of $i$ which satisfy the inequality, and hence $-1 / 2 \pm m$ has multiplicity $2 k m=(2 m(m-j)) / N$. Once again, when $m$ is 0 or 1 , this formula gives us a multiplicity of zero, as is necessary, since in row $(i i)$, the index $m$ begins at $m=2$.

We then look at the case with $m \equiv j(\bmod N), j=N / 2+2, \ldots, N-1$. Here the range $1-m<i N+\frac{N}{2} \leq m-2$ becomes

$$
j-m-\frac{N}{2} \leq i N+\frac{N}{2} \leq m-j+\frac{N}{2}
$$

by adding $j-1-\frac{N}{2}$ to the left hand side and subtracting $j-2-\frac{N}{2}$ from the right hand side. Again, we check that $0<j-1-\frac{N}{2} \leq N$, and that $0 \leq j-2-\frac{N}{2}<N$. If $m=k N+j$, where $k=0,1,2, \ldots$, then we see that there are $2 k+2$ values of $i$ which satisfy the inequality, hence $-1 / 2 \pm m$ has multiplicity $(2 k+2) m=(2 m(m-j+N)) / N$.

We can then compute the polynomials that interpolate the spectral multiplicities in the following way.

Lemma 11.9. For $m>0$, the multiplicity of $-1 / 2+m$, for $m \equiv j \bmod N$, is given by the values $P_{j}^{+}(-1 / 2+m)$ of the polynomials

$$
\begin{array}{ll}
P_{j}^{+}(u)=\frac{2}{N} u^{2}+\frac{2-2 j}{N} u+\frac{1-2 j}{2 N} & \text { for } j=0, \ldots, \frac{N}{2}+1 \\
P_{j}^{+}(u)=\frac{2}{N} u^{2}+\frac{2-2 j+2 N}{N} u+\frac{1-2 j+2 N}{2 N} & \text { for } j=\frac{N}{2}+2, \ldots, N-1 .
\end{array}
$$

For $m \geq 0$, the multiplicity of $-1 / 2-m$, for $m \equiv \ell \bmod N$, is given by the values $P_{\ell}^{-}(-1 / 2-m)$ of the polynomials

$$
\begin{array}{ll}
P_{j}^{-}(u)=\frac{2}{N} u^{2}+\frac{2+2 j}{N} u+\frac{1+2 j}{2 N} & \text { for } j=0, \ldots, \frac{N}{2}+1 \\
P_{j}^{-}(u)=\frac{2}{N} u^{2}+\frac{2+2 j-2 N}{N} u+\frac{1+2 j-2 N}{2 N}, & \text { for } j=\frac{N}{2}+2, \ldots, N-1 .
\end{array}
$$

The multiplicity of $-\left(N^{\prime}+i N\right)-\frac{1}{2}$, for $i \geq 0$, is given by the value $P^{-}\left(-\left(N^{\prime}+\right.\right.$ $i N)-1 / 2)$ of the polynomial

$$
P^{-}(u)=-2 u-1
$$

Proof. This follows directly from the expressions for the multiplicities given in Lemma 11.8 above.

We then have the following result.

Theorem 11.10. Let $f_{+}$be a Schwartz function supported on the positive reals, chosen as in Lemma 11.3, and let $D$ be an operator with spectrum given by (11.3). The spectral action is of the form

$$
\operatorname{Tr}\left(f_{+}(D / \Lambda)\right)=\frac{1}{N}\left(2 \Lambda^{3} \widehat{f}_{+}^{(2)}(0)-\Lambda^{2} \widehat{f}_{+}^{(1)}(0)-\Lambda \widehat{f}_{+}(0)\right)+O\left(\Lambda^{-k}\right)
$$


Proof. We set $g_{j}^{+}(u)=P_{j}^{+}(u) f_{+}(u / \Lambda)$, with $P_{j}^{+}$as in (11.24), for $j=$ $0, \ldots, N-1$. Then, arguing as in Theorem 11.4, we see that the spectral action is computed by

$$
\operatorname{Tr}(f(D / \Lambda))=\frac{1}{N} \sum_{j=0}^{N-1} \widehat{g}_{j}^{+}(0)+O\left(\Lambda^{-k}\right) .
$$

We can compute each term explicitly using (11.24), and we obtain

$$
\begin{aligned}
\widehat{g}_{j}^{+}(0)= & \frac{2}{N} \Lambda^{3} \widehat{f}_{+}^{(2)}(0)+\frac{2-2 j}{N} \Lambda^{2} \widehat{f}_{+}^{(1)}(0)+\frac{1-2 j}{2 N} \Lambda \widehat{f}_{+}(0) \\
& \text { for } j=0, \ldots, \frac{N}{2}+1 \\
\widehat{g}_{j}^{+}(0)= & \frac{2}{N} \Lambda^{3} \widehat{f}_{+}^{(2)}(0)+\frac{2-2 j+2 N}{N} \Lambda^{2} \widehat{f}_{+}^{(1)}(0)+\frac{1-2 j+2 N}{2 N} \Lambda \widehat{f}_{+}(0), \\
& \text { for } j=\frac{N}{2}+2 \ldots, N-1 .
\end{aligned}
$$

We have

$$
\begin{aligned}
& \sum_{j=0}^{N / 2+1} \frac{2-2 j}{N}+\sum_{j=N / 2+2}^{N-1} \frac{2-2 j+2 N}{N}=-1, \\
& \sum_{j=0}^{N / 2+1} \frac{1-2 j}{2 N}+\sum_{j=N / 2+2}^{N-1} \frac{1-2 j+2 N}{2 N}=-1 .
\end{aligned}
$$

This then gives

$$
\sum_{j=0}^{N-1} \widehat{g}_{j}^{+}(0)=2 \Lambda^{3} \widehat{f}_{+}^{(2)}(0)-\Lambda^{2} \widehat{f}_{+}^{(1)}(0)-\Lambda \widehat{f}_{+}(0) .
$$

We then proceed in a way similar to Theorem 11.5 for the case of a test function supported on the negative reals.

Theorem 11.11. Let $f_{-}$be a Schwartz function supported on the negative reals, chosen as in Lemma 11.3. The spectral action for an operator D with spectrum given by (11.3) is given by

$$
\operatorname{Tr}\left(f_{-}(D / \Lambda)\right)=\frac{1}{N}\left(2 \Lambda^{3} \widehat{f}_{-}^{(2)}(0)+3 \Lambda^{2} \widehat{f}_{-}^{(1)}(0)+\Lambda \widehat{f}_{-}(0)\right)+O\left(\Lambda^{-k}\right)
$$

Proof. We set $g_{j}^{-}(u)=P_{j}^{-}(u) f_{-}(u / \Lambda)$, with $P_{j}^{-}$as in (11.25), and $g^{-}(u)=$ $P^{-}(u) f_{-}(u / \Lambda)$, for $P^{-}$as in (11.26). By the same reasoning of Theorem 11.5 we see that, up to an error term of the order of $O\left(\Lambda^{-k}\right)$, the spectral action $\operatorname{Tr}\left(f_{-}(D / \Lambda)\right)$ is given by

$$
\frac{1}{N}\left(\widehat{g}^{-}(0)+\sum_{j=0}^{n-1} \widehat{g}_{j}^{-}(0)\right) .
$$


We then find

$$
\begin{aligned}
\widehat{g}_{j}^{-}(0)= & \frac{2}{N} \Lambda^{3} \widehat{f}_{-}^{(2)}(0)+\frac{2+2 j}{N} \Lambda^{2} \widehat{f}_{-}^{(1)}(0)+\frac{1+2 j}{2 N} \Lambda \widehat{f}_{-}(0) \\
& \text { for } j=0, \ldots, \frac{N}{2}+1 \\
\widehat{g}_{j}^{-}(0)= & \frac{2}{N} \Lambda^{3} \widehat{f}_{-}^{(2)}(0)+\frac{2+2 j-2 N}{N} \Lambda^{2} \widehat{f}_{-}^{(1)}(0)+\frac{1+2 j-2 N}{2 N} \Lambda \widehat{f}_{-}(0) \\
& \quad \text { for } j=\frac{N}{2}+2, \ldots, N-1
\end{aligned}
$$

and

We have

$$
\widehat{g}^{-}(0)=-2 \Lambda^{2} \widehat{f}_{-}^{(1)}(0)-\Lambda \widehat{f}_{-}(0) .
$$

$$
\begin{aligned}
& \sum_{j=0}^{N / 2+1} \frac{2+2 j}{N}+\sum_{j=N / 2+2}^{N-1} \frac{2+2 j-2 N}{N}-1=3 \\
& \sum_{j=0}^{N / 2+1} \frac{1+2 j}{2 N}+\sum_{j=N / 2+2}^{N-1} \frac{1+2 j-2 N}{2 N}-1=1
\end{aligned}
$$

so we obtain

$$
\widehat{g}^{-}(0)+\sum_{j=0}^{n-1} \widehat{g}_{j}^{-}(0)=2 \Lambda^{3} \widehat{f}_{-}^{(2)}(0)+3 \Lambda^{2} \widehat{f}_{-}^{(1)}(0)+\Lambda \widehat{f}_{-}(0) .
$$

We then assemble these two cases together and we obtain the following expression for the spectral action.

Theorem 11.12. Let $f$ be a Schwartz function on the real line, and let $D$ be an operator with spectrum given by (11.3). For $f_{+}$and $f_{-}$chosen as in Lemma 11.3, with $f=f_{+}+f_{-}$on an open neighborhood of the spectrum of $D$, the spectral action is given by

$$
\begin{aligned}
\operatorname{Tr}(f(D / \Lambda)) & =\frac{1}{N}\left(2 \Lambda^{3}\left(\widehat{f}_{+}^{(2)}(0)+\widehat{f}_{-}^{(2)}(0)\right)\right. \\
& \left.+\Lambda^{2}\left(3 \widehat{f}_{-}^{(1)}(0)-\widehat{f}_{+}^{(1)}(0)\right)+\Lambda\left(\widehat{f}_{-}(0)-\widehat{f}_{+}(0)\right)\right)
\end{aligned}
$$

up to an error term of the order $O\left(\Lambda^{-k}\right)$ for arbitary $k>0$.

In particular, if the function $f$ is an even function, then $\widehat{f}_{+}^{(2)}(0)=\widehat{f}_{-}^{(2)}(0)$ and $\widehat{f}_{-}(0)=\widehat{f}_{+}(0)$, while $\widehat{f}_{-}^{(1)}(0)=-\widehat{f}_{+}^{(1)}(0)$, so one obtains

$$
\operatorname{Tr}(f(D / \Lambda))=4 \Lambda^{3} \widehat{f}_{+}^{(2)}(0)-4 \Lambda^{2} \widehat{f}_{+}^{(1)}(0) .
$$

We see then that the resulting spectral action for the two spectra (11.2) and (11.3) of [3] is different, unlike what we have seen in all the other explicit cases of Dirac spectra on manifolds for which we explicitly computed the spectral action, where the spectral action is independent of the spin structure, even though the Dirac spectrum itself may be different for different 
spin structures. This is in clear contrast with the lens spaces calculation with the correct Dirac spectrum that we describe in Section 11.7, below.

However, it is interesting to notice that the two spectra (11.2) and (11.3) have the property that the spectral action computed for the operator $|D|$ instead of $D$ restores the symmetry, namely it gives the same result for the two spectra.

11.5. The spectral action for $|D|$. We consider again an operator $D$ that has as spectrum either (11.2) or (11.3). We replace $D$ by $|D|$ and we proceed to the same calculation of the spectral action as before.

Theorem 11.13. Let $D=F|D|$ be an operator with spectrum (11.2). Let $f$ be an even Schwartz function and $f_{+}$and $f_{-}$be as in Lemma 11.3, with $f=f_{+}+f_{-}$on an open neighborhood of the spectrum of $D$, and with $f_{-}(-x)=f_{+}(x)$. Then the spectral action $\operatorname{Tr}(f(|D| / \Lambda))$ is given by

$$
\operatorname{Tr}(f(|D| / \Lambda))=\frac{1}{N}\left(4 \Lambda^{3} \widehat{f}_{+}^{(2)}(0)+2 \Lambda^{2} \widehat{f}_{+}^{(1)}(0)\right)+O\left(\Lambda^{-k}\right) .
$$

Proof. Let $\lambda_{j, m}^{ \pm}= \pm m-1 / 2$ and $\lambda_{i}^{-}=-i N-1 / 2$ be the arithmetic progressions of the Dirac spectrum (11.2) on $\mathcal{L}_{N}$ with the canonical spin structure. We have obtained in Lemma 11.2 polynomials $P_{j}^{+}(u), P_{j}^{-}(u)$ and $P^{-}(u)$ such that, for $m>0, P_{j}^{+}\left(\lambda_{j, m}^{+}\right)$is the spectral multiplicity of $\lambda_{j, m}^{+}$, while for $m \leq 0, P_{j}^{-}\left(\lambda_{j, m}^{-}\right)$is the spectral multiplicity of $\lambda_{j, m}^{-}$, and $P^{-}\left(\lambda_{i}^{-}\right)$is the spectral multiplicity of $\lambda_{i}^{-}$.

When we replace $D$ by $|D|$, we want new polynomials $\bar{P}_{j}^{-}(u)$ and $\bar{P}^{-}(u)$, with the property that, for $m \leq 0, \bar{P}_{j}^{-}\left(-\lambda_{j, m}^{-}\right)$is the spectral multiplicity of $\lambda_{j, m}^{-}$and $\bar{P}^{-}\left(-\lambda_{i}^{-}\right)$is the spectral multiplicity of $\lambda_{i}^{-}$. It suffices to choose $\bar{P}_{j}^{-}(-u)=P_{j}^{-}(u)$ and $\bar{P}^{-}(-u)=P^{-}(u)$. All the polynomials $P_{j}^{+}, P_{j}^{-}$and $P^{-}$of Lemma 11.2 are of the form $c_{2} u^{2}+c_{1} u+c_{0}$ for suitable coefficients $c_{k}$. Thus, while the $P_{j}^{+}$remain the same, the corresponding $\bar{P}_{j}^{-}$and $\bar{P}^{-}$will be of the form $c_{2} u^{2}-c_{1} u+c_{0}$. More precisely, we obtain

$$
\begin{aligned}
\bar{P}_{0}^{-}(u) & =\frac{2}{N} u^{2}-\frac{2}{N} u+\frac{1}{2 N} \\
\bar{P}_{1}^{-}(u) & =\frac{2}{N} u^{2}-\frac{4}{N} u+\frac{3}{2 N} \\
\bar{P}_{j}^{-}(u) & =\frac{2}{N} u^{2}-\frac{2+2 j-N}{N} u+\frac{1+2 j-N}{2 N}, \quad j=2,3, \ldots, N-1, \\
\bar{P}^{-}(u)=2 u-1 . &
\end{aligned}
$$

Similarly, for $f$ even with $f=f_{+}+f_{-}$on an open neighborhood of the spectrum, as before, and with $f_{+}(-u)=f_{-}(u)$, we have $f_{+}\left(-\lambda_{j, m}^{-}\right)=f_{-}\left(\lambda_{j, m}^{-}\right)$ and $f_{+}\left(-\lambda_{j}^{-}\right)=f_{-}\left(\lambda_{j}^{-}\right)$.

Correspondingly, we now set

$$
g_{j}^{+}(u)=P_{j}^{+}(u) f_{+}\left(\frac{u}{\Lambda}\right), \quad \bar{g}_{j}^{-}(u)=\bar{P}_{j}^{-}(u) f_{-}\left(\frac{u}{\Lambda}\right), \quad \bar{g}^{-}(u)=\bar{P}^{-}(u) f_{-}\left(\frac{u}{\Lambda}\right),
$$


We see then that $\operatorname{Tr}(f(|D| / \Lambda))$ is given by (11.36)

$$
\sum_{k \in \mathbb{Z}}\left(\sum_{j=0}^{N-1} g_{j}^{+}\left(k N+\frac{2 j-1}{2}\right)+\sum_{j=0}^{N-1} \bar{g}_{j}^{-}\left(k N+\frac{2 j+1}{2}\right)+\bar{g}^{-}\left(k N+\frac{1}{2}\right)\right) .
$$

We then use Poisson summation as before and we find

$$
\operatorname{Tr}(f(|D| / \Lambda))=\frac{1}{N}\left(\sum_{j=0}^{N-1} \widehat{g}_{j}^{+}(0)+\sum_{j=0}^{N-1} \widehat{\bar{g}_{j}^{-}}(0)+\widehat{\bar{g}^{-}}(0)\right)+O\left(\Lambda^{-k}\right) .
$$

We then see that

$$
\sum_{j=0}^{N-1} \widehat{g}_{j}^{+}(0)=2 \Lambda^{3} \widehat{f}_{+}^{(2)}(0)+\Lambda^{2} \widehat{f}_{+}^{(1)}(0)
$$

as before, while

$$
\sum_{j=0}^{N-1} \widehat{\bar{g}_{j}^{-}}(0)+\widehat{\bar{g}^{-}}(0)=2 \Lambda^{3} \widehat{f}_{-}^{(2)}(0)-\Lambda^{2} \widehat{f}_{-}^{(1)}(0)=2 \Lambda^{3} \widehat{f}_{+}^{(2)}(0)+\Lambda^{2} \widehat{f}_{+}^{(1)}(0),
$$

so that (11.32) holds.

We now see that this is the same result obtained from the second spectrum (11.3).

Theorem 11.14. Let $D=F|D|$ be an operator with spectrum (11.3). Let $f$ be an even Schwartz function and $f_{+}$and $f_{-}$be as in Lemma 11.3, with $f=f_{+}+f_{-}$on an open neighborhood of the spectrum of $D$, and with $f_{-}(-x)=f_{+}(x)$. Then the spectral action $\operatorname{Tr}(f(|D| / \Lambda))$ is given by

$$
\operatorname{Tr}(f(|D| / \Lambda))=\frac{1}{N}\left(4 \Lambda^{3} \widehat{f}_{+}^{(2)}(0)+2 \Lambda^{2} \widehat{f}_{+}^{(1)}(0)\right)+O\left(\Lambda^{-k}\right) .
$$

Proof. The argument is the same as in the previous case, but applied to the eigenvalues and multiplicities (11.3) and the polynomials $P_{j}^{+}, P_{j}^{-}$and $P^{-}$of Lemma 11.9, We then compute $\operatorname{Tr}(f(|D| / \Lambda))$ as in the case of the canonical spin structure, using the corresponding polynomials $\bar{P}_{j}^{-}$and $\bar{P}^{-}$ and the functions $\bar{g}_{j}^{-}$and $\bar{g}^{-}$as above. We obtain again the expression (11.37), where in this case

$$
\sum_{j=0}^{N-1} \widehat{g}_{j}^{+}(0)=2 \Lambda^{3} \widehat{f}_{+}^{(2)}(0)-\Lambda^{2} \widehat{f}_{+}^{(1)}(0)-\Lambda \widehat{f}_{+}(0)
$$

and

$$
\begin{aligned}
\sum_{j=0}^{N-1} \widehat{\bar{g}_{j}^{-}}(0)+\widehat{\bar{g}^{-}}(0) & =2 \Lambda^{3} \widehat{f}_{-}^{(2)}(0)-3 \Lambda^{2} \widehat{f}_{-}^{(1)}(0)+\Lambda \widehat{f}_{-}(0) \\
& =2 \Lambda^{3} \widehat{f}_{+}^{(2)}(0)+3 \Lambda^{2} \widehat{f}_{+}^{(1)}(0)+\Lambda \widehat{f}_{+}(0)
\end{aligned}
$$


so that $\operatorname{Tr}(f(|D| / \Lambda))$ is given by

$$
\frac{1}{N}\left(2 \Lambda^{3} \widehat{f}_{+}^{(2)}(0)-\Lambda^{2} \widehat{f}_{+}^{(1)}(0)-\Lambda \widehat{f}_{+}(0)+2 \Lambda^{3} \widehat{f}_{+}^{(2)}(0)+3 \Lambda^{2} \widehat{f}_{+}^{(1)}(0)+\Lambda \widehat{f}_{+}(0)\right),
$$

up to an error term of the order of $O\left(\Lambda^{-k}\right)$. This gives again the same (11.38) as for the canonical spin structure.

11.6. The slow-roll potential: a false positive. Now we use this result to compute the spectral action for an operator $\mathcal{D}^{2}$ with

$$
\mathcal{D}=\left(\begin{array}{cc}
0 & D \otimes 1+i \otimes D_{S^{1}} \\
D \otimes 1-i \otimes D_{S^{1}} & 0
\end{array}\right)
$$

where $D_{S^{1}}$ has spectrum $\beta^{-1}(\mathbb{Z}+1 / 2)$, and $D$ is an operator with spectrum either (11.2) or (11.3).

The spectrum of the operator $\mathcal{D}^{2}$ will be contained in the set of values of the form $\left(\lambda_{j, m}^{ \pm}\right)^{2}(\Lambda a)^{-2}+\lambda_{n}^{2}(\Lambda \beta)^{-2}$ and $\left(\lambda_{i}^{-}\right)^{2}(\Lambda a)^{-2}+\lambda_{n}^{2}(\Lambda \beta)^{-2}$, where $\lambda_{j, m}^{ \pm}$and $\lambda_{i}^{-}$are the arithmetic progressions associated to the spectrum of $D$ and $\lambda_{n}=n+1 / 2$ are the eigenvalues on a circle of radius one. We see that the pairs of points $(u, v)$ in $\mathbb{R}^{2}$ which are of the form $\left(\lambda_{j, m}^{ \pm}, \lambda_{n}\right)$ or $\left(\lambda_{i}^{-}, \lambda_{n}\right)$ all lie outside of a vertical strip around $u=0$. We fix a value $\alpha<1$ such that the strip $u \in(-\alpha, \alpha)$ contains on such pair. We also fix a $k>0$, which will determine the order $O\left(\Lambda^{-k}\right)$ of error in the spectral action computation. Let then $\ell\left(u^{k}\right)$ be a smooth function, which is equal to zero for $u \leq 0$ and is equal to one for $u \geq \alpha$.

Lemma 11.15. Suppose given a polynomial $P(x)=c_{2} x^{2}+c_{1} x+c_{0}$, and let $h$ be a Schwartz function on $\mathbb{R}$. The difference between the integrals

$$
I_{1}=\int_{\mathbb{R}^{2}} P(x) \ell\left(x^{k}(\Lambda a)^{k}\right) h\left(x^{2}+y^{2}\right) d x d y
$$

and

$$
I_{2}=\int_{0}^{\infty} \int_{-\pi / 2}^{\pi / 2}\left(\frac{c_{2}}{2} \rho^{2}+c_{1} \rho \cos \theta+c_{2}\right) h\left(\rho^{2}\right) \rho d \rho d \theta
$$

is bounded by

$$
\left|I_{1}-I_{2}\right|=O\left(\Lambda^{-k}\right)
$$

Proof. The difference $I_{1}-I_{2}$ is computed by

$$
\left|\int_{\mathbb{R}}\left(\int_{0}^{\alpha} P(x) \ell\left(x^{k}(\Lambda a)^{k}\right) h\left(x^{2}+y^{2}\right) d x\right) d y\right| \leq C \frac{\alpha^{k}}{(\Lambda a)^{k}} .
$$

We can then proceed to compute the spectral action. 
Theorem 11.16. The spectral action for the operator $\mathcal{D}$ of (11.39), where $D$ has spectrum (11.2) is of the form

$\operatorname{Tr}\left(h\left(\mathcal{D}^{2} / \Lambda^{2}\right)\right)=2 \pi \Lambda^{4} a^{3} \beta \int_{0}^{\infty} u h(u) d u+2 \Lambda^{3} a^{2} \beta \int_{0}^{\infty} u^{1 / 2} h(u) d u+O\left(\Lambda^{-k}\right)$.

Proof. For a given $k>0$, we choose a cutoff $\ell\left(u^{k}\right)$ as in Lemma 11.15. We then set $\ell_{+}(u)=\ell(u)$ and $\ell_{-}(u)=\ell_{+}(-u)$. We then consider, for $j=0, \ldots N-1$, functions of the form

$$
g_{j}^{+}(u, v)=2 P_{j}^{+}(u) \ell_{+}\left(u^{k}\right) h\left(u^{2}(\Lambda a)^{-2}+v^{2}(\Lambda \beta)^{-2}\right),
$$

where the polynomials $P_{j}^{+}(u)$ are as in (11.5). We also set

$$
g_{j}^{-}(u, v)=2 \bar{P}_{j}^{-}(u) \ell_{-}\left(u^{k}\right) h\left(u^{2}(\Lambda a)^{-2}+v^{2}(\Lambda \beta)^{-2}\right),
$$

with the polynomials $\bar{P}_{j}^{-}(u)$ of (11.33) and

$$
g^{-}(u, v)=2 \bar{P}^{-}(u) \ell_{-}\left(u^{k}\right) h\left(u^{2}(\Lambda a)^{-2}+v^{2}(\Lambda \beta)^{-2}\right),
$$

with $\bar{P}^{-}(u)$ as in (11.34). The spectral action $\operatorname{Tr}\left(h\left(\mathcal{D}^{2} / \Lambda^{2}\right)\right)$, for $D$ with spectrum (11.2), is then given by

$$
\begin{aligned}
\operatorname{Tr}\left(h\left(\mathcal{D}^{2} / \Lambda^{2}\right)\right) & =\sum_{j=0}^{N-1} g_{j}^{+}\left(n N+\frac{(2 j-1)}{2}, m+\frac{1}{2}\right) \\
& +\sum_{j=0}^{N-1} g_{j}^{-}\left(n N-\frac{(2 j+1)}{2}, m+\frac{1}{2}\right) \\
& +g^{-}\left(n N-\frac{1}{2}, m+\frac{1}{2}\right) .
\end{aligned}
$$

We compute it by applying the Poisson summation formula to the functions (11.44), (11.45), and (11.46). We obtain, as in the previous cases

$$
\operatorname{Tr}\left(h\left(\mathcal{D}^{2} / \Lambda^{2}\right)\right)=\frac{1}{N}\left(\sum_{j=0}^{N-1} \widehat{g}_{j}^{+}(0,0)+\sum_{j=0}^{N-1} \widehat{g}_{j}^{-}(0,0)+\widehat{g}^{-}(0,0)\right)+O\left(\Lambda^{-k}\right) .
$$

We then use Lemma 11.15 to estimate the integrals, up to an error term of order $O\left(\Lambda^{-k}\right)$, to be of the form

$$
\begin{aligned}
\sum_{j=0}^{N-1} \widehat{g}_{j}^{+}(0,0) & =2 \Lambda^{2} a \beta \int_{0}^{\infty} \int_{-\pi / 2}^{\pi / 2}\left(\rho^{2}(\Lambda a)^{2}+\rho \cos \theta(\Lambda a)\right) h\left(\rho^{2}\right) \rho d \rho d \theta \\
& =2 \pi \Lambda^{4} a^{3} \beta \int_{0}^{\infty} \rho^{3} h\left(\rho^{2}\right) d \rho+2 \Lambda^{3} a^{2} \beta \int_{0}^{\infty} \rho^{2} h\left(\rho^{2}\right) d \rho
\end{aligned}
$$

where we used the fact that

$$
\sum_{j=0}^{N-1} P_{j}^{+}(u)=2 u^{2}+u
$$


After a change of variables, we write the above as

$$
\sum_{j=0}^{N-1} \widehat{g}_{j}^{+}(0,0)=\pi \Lambda^{4} a^{3} \beta \int_{0}^{\infty} u h(u) d u+\Lambda^{3} a^{2} \beta \int_{0}^{\infty} u^{1 / 2} h(u) d u .
$$

Similarly, using the approximation of Lemma 11.15 we obtain, up to an error term of the order of $O\left(\Lambda^{-k}\right)$,

$$
\begin{aligned}
\sum_{j=0}^{N-1} \widehat{g}_{j}^{-}(0,0)+\widehat{g}^{-}(0,0) & =2 \pi \Lambda^{4} a^{3} \beta \int_{0}^{\infty} \rho^{3} h\left(\rho^{2}\right) d \rho+2 \Lambda^{3} a^{2} \beta \int_{0}^{\infty} \rho^{2} h\left(\rho^{2}\right) d \rho \\
& =\pi \Lambda^{4} a^{3} \beta \int_{0}^{\infty} u h(u) d u+\Lambda^{3} a^{2} \beta \int_{0}^{\infty} u^{1 / 2} h(u) d u
\end{aligned}
$$

where we used the fact that

$$
\sum_{j=0}^{N-1} \bar{P}_{j}^{-}(u)+\bar{P}^{-}(u)=2 u^{2}-u
$$

and that $\ell_{-}(u)=\ell_{+}(-u)$. This then gives (11.43).

The case where $D$ has spectrum (11.3) is analogous and yields the same result.

Theorem 11.17. Consider the operator $\mathcal{D}$ of (11.39), where $D$ has spectrum (11.3). The spectral action is of the form

$\operatorname{Tr}\left(h\left(\mathcal{D}^{2} / \Lambda^{2}\right)\right)=2 \pi \Lambda^{4} a^{3} \beta \int_{0}^{\infty} u h(u) d u+2 \Lambda^{3} a^{2} \beta \int_{0}^{\infty} u^{1 / 2} h(u) d u+O\left(\Lambda^{-k}\right)$.

Proof. One proceeds exactly as in Theorem 11.16, but using the expressions for $P_{j}^{+}, \bar{P}_{j}^{-}$and $\bar{P}^{-}$as in Theorem 11.14. One then has

$$
\sum_{j=0}^{N-1} P_{j}^{+}(u)=2 u^{2}-u-1, \quad \sum_{j=0}^{N-1} \bar{P}_{j}^{-}(u)+P^{-}(u)=2 u^{2}-3 u+1,
$$

so that, using $\ell_{-}(u)=\ell_{+}(-u)$, one correspondingly obtains

$$
\begin{aligned}
\sum_{j=0}^{N-1} \widehat{g}_{j}^{+}(0,0) & =2 \pi \Lambda^{4} a^{3} \beta \int_{0}^{\infty} \rho^{3} h\left(\rho^{2}\right) d \rho-2 \Lambda^{3} a^{2} \beta \int_{0}^{\infty} \rho^{2} h\left(\rho^{2}\right) d \rho \\
& -2 \pi \Lambda^{2} a \beta \int_{0}^{\infty} h\left(\rho^{2}\right) \rho d \rho \\
& =\pi \Lambda^{4} a^{3} \beta \int_{0}^{\infty} u h(u) d u-\Lambda^{3} a^{2} \beta \int_{0}^{\infty} u^{1 / 2} h(u) d u \\
& -\pi \Lambda^{2} a \beta \int_{0}^{\infty} h(u) d u .
\end{aligned}
$$


Similarly, one obtains

$$
\begin{aligned}
\sum_{j=0}^{N-1} \widehat{g}_{j}^{-}(0,0)+\widehat{g}^{-}(0,0) & =\pi \Lambda^{4} a^{3} \beta \int_{0}^{\infty} u h(u) d u \\
& +3 \Lambda^{3} a^{2} \beta \int_{0}^{\infty} u^{1 / 2} h(u) d u \\
& +\pi \Lambda^{2} a \beta \int_{0}^{\infty} h(u) d u .
\end{aligned}
$$

Thus, adding these contributions one obtains then the same (11.51) as in the previous case.

We then see that the form of the associated potential $V(\phi)$ coming from the perturbations $\mathcal{D}^{2} \mapsto \mathcal{D}^{2}+\phi^{2}$ is very different from the 3 -sphere and the other spherical manifolds computed in this paper, quaternionic and dodecahedral space.

We set

$\mathcal{V}(x)=\int_{0}^{\infty} u(h(u+x)-h(u)) d u, \quad \mathcal{Z}(x)=\int_{0}^{\infty} u^{1 / 2}(h(u+x)-h(u)) d u$, in the variable $x=\phi^{2} / \Lambda^{2}$.

Proposition 11.18. Let $\mathcal{D}$ be the operator of (11.39). We have (11.55)

$\operatorname{Tr}\left(h\left(\left(\mathcal{D}^{2}+\phi^{2}\right) / \Lambda^{2}\right)\right)=\operatorname{Tr}\left(h\left(\mathcal{D}^{2} / \Lambda^{2}\right)\right)+2 \pi \Lambda^{4} a^{3} \beta \mathcal{V}\left(\phi^{2} / \Lambda^{2}\right)+2 \Lambda^{3} a^{2} \beta \mathcal{Z}\left(\phi^{2} / \Lambda^{2}\right)$.

Thus, the potential $V(x)$, for $x=\phi^{2} / \Lambda^{2}$ is of the form

$$
V(x)=2 \pi \Lambda^{4} a^{3} \beta \mathcal{V}\left(\phi^{2} / \Lambda^{2}\right)+2 \Lambda^{3} a^{2} \beta \mathcal{Z}\left(\phi^{2} / \Lambda^{2}\right) .
$$

Proof. This is an immediate consequence of Theorems 11.16 and 11.17 .

We then see that the form of the slow-roll parameters is also different in this case.

Proposition 11.19. The slow-roll parameters for the potential $V(x)$ are given by

$$
\epsilon(x)=\frac{m_{P l}^{2}}{8 \pi} A, \quad \text { and } \quad \eta(x)=\frac{m_{P l}^{2}}{8 \pi}(B-A),
$$

where

$$
\begin{gathered}
A=\frac{1}{2}\left(\frac{\pi C \mathcal{V}^{\prime}(x)+\mathcal{Z}^{\prime}(x)}{\pi C \mathcal{V}(x)+\mathcal{Z}(x)}\right)^{2} \\
B=\frac{\pi C \mathcal{V}^{\prime \prime}(x)+\mathcal{Z}^{\prime \prime}(x)}{\pi C \mathcal{V}(x)+\mathcal{Z}(x)} .
\end{gathered}
$$

Proof. This follows directly from the definition of the slow-roll parameters, having imposed the condition $\Lambda(t) \sim 1 / a(t)$, so that $\Lambda a=C$, on the Friedmann form of the spacetime back in Lorentzian signature. 
This calculation creates a "false positive" which gives the impression that there are spherical manifolds for which the inflation potential and slow-roll parameters are genuinely different from those of the sphere. This would make for a much stronger correlation between inflation and cosmic topology than what we have observed in the previous section, with different inflation scenarios not only between spherical and flat cases, but even between different topologies with the same underlying spherical geometry. However, this turns out not to be the case. The true story of the lens spaces, described in the coming section, shows that in fact, with the correct calculation of the Dirac spectrum, they behave exactly as the other spherical topology, with the same slow-roll parameters as in the simply connected case.

11.7. Lens spaces: a discrepancy. In this section we compute the Dirac spectrum for lens space using the same generating function technique due to Bär [2], that we used to compute the Dirac spectrum for the Poincaré homology sphere, and compare the results to the calculation in [3].

For simplicity, let us just consider the space $\mathcal{L}_{N}=S U(2) / \mathbb{Z}_{N}$ in the case $N=4$ with the canonical spin structure. By applying equations (7.4), (7.5), one obtains that the generating functions for the spectral multiplicities for $\mathcal{L}_{4}$, with the canonical spin structure, are given by:

$$
\begin{aligned}
& F_{+}(z)=-\frac{2\left(z+5 z^{3}+z^{5}+z^{7}\right)}{\left(-1+z^{2}\right)^{3}\left(1+z^{2}\right)^{2}}, \\
& F_{-}(z)=-\frac{2\left(1+z^{2}+5 z^{4}+z^{6}\right)}{\left(-1+z^{2}\right)^{3}\left(1+z^{2}\right)^{2}} .
\end{aligned}
$$

Proceeding in precisely the same manner as in the case of the Poincaré homology sphere, one obtains the following lemma.

Lemma 11.20. There are polynomials $P_{k}(u)$, for $k=0, \ldots, 3$, so that $P_{k}(3 / 2+k+4 j)=m(3 / 2+k+4 j, D)$ for all $j \in \mathbb{Z}$. The $P_{k}(u)$ are given as follows:

$$
\begin{aligned}
P_{k} & =0, \quad \text { whenever } k \text { is even. } \\
P_{1}(u) & =\frac{1}{8}-\frac{1}{2} u+\frac{1}{2} u^{2} . \\
P_{3}(u) & =-\frac{3}{8}+\frac{1}{2} u+\frac{1}{2} u^{2} .
\end{aligned}
$$

Before comparing these multiplicities with the ones given in [3], let us first compute the nonperturbative spectral action of the lens space.

Theorem 11.21. Let $D$ be the Dirac operator on $\mathcal{L}_{4}$, with the canonical spin structure. Then, for $f$ a Schwartz function, the spectral action is given 
by

$$
\operatorname{Tr}(f(D / \Lambda))=\frac{1}{4}\left(\Lambda^{3} \widehat{f}^{(2)}(0)-\frac{1}{4} \Lambda \widehat{f}(0)\right),
$$

which is precisely $1 / 4$ of the spectral action on the sphere.

Proof. As usual the result follows by applying Poisson summation to the functions $g_{j}(u)=P_{j}(u) f(u / \Lambda)$. This gives, up to an error term which is of the order of $O\left(\Lambda^{-k}\right)$ for any $k>0$, the spectral action in the form

$$
\operatorname{Tr}(f(D / \Lambda))=\frac{1}{4} \sum_{j=0}^{3} \widehat{g}_{j}(0)=\frac{1}{4} \int_{\mathbb{R}} \sum_{j} P_{j}(u) f(u / \Lambda) d u .
$$

It suffices then to notice that

$$
\sum_{j=0}^{3} P_{j}(u)=u^{2}-\frac{1}{4}
$$

The result then follows as in the sphere case.

Observe that this time around, the spectral action is a constant multiple of the spectral action of the sphere, and so one obtains the same slow-roll parameters as in the simply connected case, just as with the other spherical space forms.

Let us compare the multiplicities obtained using the generation function method in Lemma 11.20 with those obtained using the results of [3] in Lemma 11.2. By setting $N=4$ in Lemma 11.2 it is immediately evident that the two sets of multiplicities do not agree.

As a side remark, even if we replace $-(m+1)<i N$ with $-(m-2) \leq i N$ in equation (11.2) when performing the computation of Lemma 11.2, this just results in altering $P_{0}^{ \pm}$, and $P_{1}^{ \pm}$very slightly, while leaving the other $P_{j}^{ \pm}$unchanged, and the resulting multiplicities still do not agree with the multiplicities of Lemma 11.20.

We are inclined to believe that the generating function method of Lemma 11.20 gives the correct answer because of two reasons. First, the generating function method leads to a spectral action of $1 /|G|$ times the spectral action of $S^{3}$ where $G$ is the group acting on $S^{3}$, and this is exactly the result we obtained for the other spherical space forms. Secondly, if one computes the Dirac spectrum of $S U(2) / Q 8$ using the generating function method, one gets the same answer as obtained by Ginoux in [17, where the Dirac spectrum of $S U(2) / Q 8$ is computed using representation theoretic methods.

\section{REFERENCES}

[1] R. Aurich, S. Lustig, F. Steiner, H. Then, Cosmic microwave background alignment in multi-connected universes, Class. Quantum Grav. 24 (2007) 1879-1894.

[2] C. Bär, The Dirac operator on space forms of positive curvature, J. Math. Soc. Japan, 48 (1996) N.1, 69-83. 
[3] C. Bär, The Dirac operator on homogeneous spaces and its spectrum on 3-dimensional lens spaces, Arch. Math. Vol.59 (1992) 65-79.

[4] C. Bär, Dependence of Dirac Spectrum on the Spin Structure, Séminaires \& Congrès, 4, 2000, 17-33.

[5] P. de Bernardis, P.A.R. Ade, J.J. Bock, J.R. Bond, J. Borrill, A. Boscaleri, K. Coble, B.P. Crill, G.De Gasperis, P.C. Farese, P.G. Ferreira, K. Ganga, M. Giacometti, E. Hivon, V.V. Hristov, A. Iacoangeli, A.H. Jaffe, A.E. Lange, L. Martinis, S. Masi, P.V. Mason, P.D. Mauskopf, A. Melchiorri, L. Miglio, T. Montroy, C.B. Netterfield, E. Pascale, F. Piacentini, D. Pogosyan, S. Prunet, S. Rao, G. Romeo, J.E. Ruhl, F. Scaramuzzi, D. Sforna, N. Vittorio, A flat Universe from high-resolution maps of the cosmic microwave background radiation, Nature 404 (2000), 955-959.

[6] T. van den Broek, W.D. van Suijlekom, Supersymmetric QCD and noncommutative geometry, arXiv:1003.3788.

[7] S. Caillerie, M. Lachièze-Rey, J.P. Luminet, R. Lehoucq, A. Riazuelo, J. Weeks, A new analysis of the Poincaré dodecahedral space model, Astronomy and Astrophysics, Vol.476 (2007) N.2, 691-696.

[8] A. Chamseddine, A. Connes, The spectral action principle. Comm. Math. Phys. 186 (1997), no. 3, 731-750.

[9] A. Chamseddine, A. Connes, The uncanny precision of the spectral action, Commun. Math. Phys. 293 (2010) 867-897.

[10] A. Chamseddine, A. Connes, M. Marcolli, Gravity and the standard model with neutrino mixing, Adv. Theor. Math. Phys. 11 (2007), no. 6, 991-1089.

[11] A. Connes, Gravity coupled with matter and foundation of noncommutative geometry. Commun. Math. Phys., 182 (1996) 155-176.

[12] N.J. Cornish, D.N. Spergel, G.D. Starkman, E. Komatsu, Constraining the topology of the universe, Phys. Rev. Lett. 92 (2004) 201302 [4 pages].

[13] M. Dahl, Prescribing eigenvalues of the Dirac operator, Manuscripta Math. 118 (2005) 191-199.

[14] M. Dahl, Dirac eigenvalues for generic metrics on three-manifolds, Annals of Global Analysis and Geometry, 24 (2003) 95-100.

[15] A. De Simone, M.P. Hertzberg, F. Wilczek, Running inflation in the Standard Model, arXiv:0812.4946v2.

[16] E. Gausmann, R. Lehoucq, J.P. Luminet, J.P. Uzan, J. Weeks, Topological lensing in spherical spaces, Class. Quantum Grav. 18 (2001) 5155-5186.

[17] N. Ginoux, The spectrum of the Dirac operator on $\mathrm{SU}_{2} / \mathrm{Q}_{8}$. Manuscripta Math. 125 (2008), no. 3, 383-409.

[18] G.I. Gomero, M.J. Reboucas, R. Tavakol, Detectability of cosmic topology in almost flat universes, Class. Quant. Grav. 18 (2001) 4461-4476.

[19] G.I. Gomero, M.J. Reboucas, A.F.F. Teixeira, Spikes in cosmic crystallography II: topological signature of compact flat universes, Phys. Lett. A 275 (2000) 355-367.

[20] N. Hitchin, Harmonic spinors, Advances Math. 14 (1974) 1-55.

[21] M. Kamionkowski, D.N. Spergel, N. Sugiyama, Small-scale cosmic microwave background anisotropies as a probe of the geometry of the universe, Astrophysical J. 426 (1994) L 57-60.

[22] M. Lachièze-Rey, J.P. Luminet, Cosmic topology. Physics Reports, 254 (1995) 135214.

[23] R. Lehoucq, J. Weeks, J.P. Uzan, E. Gausmann, J.P. Luminet, Eigenmodes of threedimensional spherical spaces and their applications to cosmology. Class. Quantum Grav. 19 (2002) 4683-4708.

[24] J.P. Luminet, J. Weeks, A. Riazuelo, R. Lehoucq, Dodecahedral space topology as an explanation for weak wide-angle temperature correlations in the cosmic microwave background, Nature 425 (2003) 593-595. 
[25] M. Marcolli, E. Pierpaoli, Early universe models from noncommutative geometry, arXiv:0908.3683

[26] B. McInnes, APS instability and the topology of the brane-world. Physics Letters B, Vol.593 (2004) N.1-4, 10-16.

[27] W. Nelson, M. Sakellariadou, Natural inflation mechanism in asymptotic noncommutative geometry, Phys. Lett. B (2009) Vol.680, 263-266.

[28] A. Niarchou, A. Jaffe, Imprints of spherical nontrivial topologies on the cosmic microwave background, Physical Review Letters, 99 (2007) 081302, 4pp.

[29] A. de Oliveira-Costa, M. Tegmark, M. Zaldarriaga, A. Hamilton, Significance of the largest scale CMB fluctuations in WMAP, Phys. Rev. D 69 (2004) 063516 [12 pages].

[30] F. Pfäffle, The Dirac spectrum of Bieberbach manifolds, J. Geom. Phys. 35 (2000) $367-385$.

[31] A. Riazuelo, J.P. Uzan, R. Lehoucq, J. Weeks, Simulating Cosmic Microwave Background maps in multi-connected spaces, Phys.Rev. D69 (2004) 103514 [28 pages].

[32] A. Riazuelo, J. Weeks, J.P. Uzan, R. Lehoucq, J.P. Luminet, Cosmic microwave background anisotropies in multiconnected flat spaces, Phys. Rev. D 69 (2004) 103518 [25 pages].

[33] B.F. Roukema, P.T. Rózański, The residual gravity acceleration effect in the Poincaré dodecahedral space, Astronomy and Astrophysics 502 (2009) 27 [11 pages]

[34] T. Souradeep, A. Hajian, Statistical isotropy of CMB anisotropy from WMAP, arXiv:astro-ph/0502248

[35] D.N. Spergel, L. Verde, H.V. Peiris, E. Komatsu, M.R. Nolta, C.L. Bennett, M. Halpern, G. Hinshaw, N. Jarosik, A. Kogut, M. Limon, S.S. Meyer, L. Page, G.S. Tucker, J.L. Weiland, E. Wollack, E.L. Wright, First year Wilkinson Microwave Anisotropy Probe (WMAP) observations: determination of cosmological parameters, Astrophys. J. Suppl. 148 (2003) 175-194.

[36] M. Tegmark, A. de Oliveira-Costa, A. Hamilton, A high resolution foreground cleaned CMB map from WMAP, Phys. Rev. D. 68 (2003) 123523.

[37] J.P. Uzan, U. Kirchner, Ulrich, G.F.R. Ellis, WMAP data and the curvature of space, Mon. Not. Roy. Astron. Soc. 344 (2003) L65.

[38] J.P. Uzan, A. Riazuelo, R. Lehoucq, J. Weeks, Cosmic microwave background constraints on lens spaces. Phys. Rev. D, 69 (2004), 043003, 4 pp.

[39] J. Weeks, J. Gundermann, Dodecahedral topology fails to explain quadrupole-octupole alignment, Class. Quantum Grav. 24 (2007) 1863-1866.

[40] J. Weeks, R. Lehoucq, J.P. Uzan, Detecting topology in a nearly flat spherical universe, Class. Quant. Grav. 20 (2003) 1529-1542.

Department of Mathematics, California Institute of Technology, Pasadena, CA 91125, USA

E-mail address: matilde@caltech.edu

E-mail address: teh@caltech.edu

Department of Physics and Astronomy, University of Southern California, Los Angeles, CA 90089, USA

E-mail address: pierpaol@usc.edu 